\title{
Eruptions and flaring activity in emerging quadrupolar regions
}

\author{
P. Syntelis, E. J. Lee, C. W. Fairbairn, V. Archontis, and A. W. Hood
}

\author{
School of Mathematics and Statistics, University of St Andrews, St Andrews, Fife KY16 9SS, UK \\ e-mail: ps84@st-andrews.ac.uk
}

Received 5 July 2019 / Accepted 3 September 2019

\begin{abstract}
Context. Solar observations suggest that some of the most dynamic active regions are associated with complex photospheric magnetic configurations such as quadrupolar regions, and especially those that have a $\delta$-spot configuration and a strong polarity inversion line (PIL).

Aims. We study the formation and eruption of magnetic flux ropes in quadrupolar regions.

Methods. We performed 3D magnetohydrodynamics simulations of the partial emergence of a highly twisted flux tube from the solar interior into a non-magnetised stratified atmosphere. We introduced a density deficit at two places along the length of the subphotospheric flux tube to emerge as two $\Omega$-shaped loops, forming a quadrupolar region.

Results. At the photosphere, the emerging flux forms two initially separated bipoles, which later come in contact, forming a $\delta$-spot central region. Above the two bipoles, two magnetic lobes expand and interact through a series of current sheets at the interface between them. Two recurrent confined eruptions are produced. In both cases, the reconnection between sheared low-lying field lines forms a flux rope. The reconnection between the two lobes higher in the atmosphere forms field lines that retract down and push against the flux rope, creating a current sheet between them. It also forms field lines that create a third magnetic lobe between the two emerged lobes, that later acts as a strapping field. The flux rope eruptions are triggered when the reconnection between the flux ropes and the field above the ropes becomes efficient enough to remove the tension of the overlying field. These reconnection events occur internally in the quadrupolar system, as the atmosphere is non-magnetised. The flux rope of the first, weaker, eruption almost fully reconnects with the overlying field. The flux rope of the second, more energetic, eruption is confined by the overlying strapping field. During the second eruption, the flux rope is enhanced in size, flux, and twist, similar to confined-flare-to-flux-rope observations. Proxies of the emission reveal the two erupting filaments channels. A flare arcade is only formed in the second eruption owing to the longer lasting and more efficient reconnection at the current sheet below the flux rope.
\end{abstract}

Key words. Sun: activity - Sun: corona - Sun: flares - Sun: magnetic fields - magnetohydrodynamics (MHD) - methods: numerical

\section{Introduction}

Flux emergence is a key process that transfers magnetic energy from the solar interior, through the photosphere, up into the solar corona. It has long been posited that the buoyant rise of magnetic flux tubes could be responsible for the production of active regions (Parker 1955). Active regions are highly associated with a plethora of solar phenomena, such as flares and coronal mass ejections (CMEs), whereby stored free magnetic energy is released in an explosive manner (e.g. Priest \& Forbes 2002). The amount of stored and released energy can range significantly depending on the specifics of the active regions, leading to more or less energetic eruptions or flares.

The most intense solar phenomena are commonly associated with the presence of a strong, i.e. high-gradient, polarity inversion line (PIL; e.g. Schrijver 2009). It is no surprise, therefore, that large active regions with strong PILs, such as NOAA 11429 (e.g. Liu et al. 2014a; Wang et al. 2014; Chintzoglou et al. 2015; Syntelis et al. 2016; Patsourakos et al. 2016; Zheng et al. 2017; Yang et al. 2018; Baker et al. 2019; Zhou et al. 2019) and the quadrapolar NOAA 11158 (e.g. Schrijver et al. 2011; Sun et al. 2012; Wang et al. 2012; Liu et al. 2012; Vemareddy et al. 2012; Dalmasse et al. 2013; Tziotziou et al. 2013; Janvier et al. 2014; Kazachenko et al. 2015; Zhang et al. 2016) have been extensively studied as a consequence of the strong activity occurring during their long lifetimes. Prominent examples where strong PILs are commonly found are the so-called $\delta$-spots (Künzel 1960).
These are regions in which the two opposite polarities are so compact that in white light observations the umbra of the two polarities share a common penumbra. The $\delta$-spots are highly associated with intense solar activity and strong X-class flares (e.g. Zirin \& Liggett 1987; Schrijver 2007; Guo et al. 2014). Strong PILs and $\delta$-spots can develop in a variety of different photospheric magnetic configurations. For instance, they can be formed between the two opposite polarities in a bipolar region, between the innermost polarities of a quadrupolar region, between the main polarity of an existing region and a parasitic polarity, between polarities of very complex shape, or even between polarities of separate active regions (e.g. Linton et al. 1999; Fan et al. 1999; Takasao et al. 2015; Fang \& Fan 2015; Toriumi et al. 2014, 2017; Toriumi \& Takasao 2017; Knizhnik et al. 2018; Chintzoglou et al. 2019).

The strong shearing along such strong PILs, the rotation of the polarities, and/or the direct emergence of a non-potential field inject shear into the solar atmosphere, building up free magnetic energy, part of which accumulates along the PIL. This free energy is usually stored either in a sheared magnetic arcade (e.g. Antiochos et al. 1999; Lynch et al. 2008) or a magnetic flux rope (e.g. van Ballegooijen \& Martens 1989; Török \& Kliem 2005). When such configurations are destabilised, they release part of their free energy in the form of a flare arcade and an accelerating magnetic flux rope (either a pre-existing or formed on-the-fly) whose flux and twist are enhanced during the eruption 
(e.g. Inoue et al. 2018; Syntelis et al. 2019a). However, such events are not always associated with the runaway ejection of plasma such as a CME. Depending on the specifics of the eruption, the erupting field can move upwards without being stopped (ejective eruption and eruptive flare; e.g. Qiu \& Yurchyshyn 2005; Vourlidas et al. 2012; Mitra et al. 2018; Georgoulis et al. 2019), or it can be decelerated and become confined by an overlying strapping field (confined eruption and flare; e.g. Moore et al. 2001; Patsourakos et al. 2013; Liu et al. 2018).

In order for an ejective eruption to occur, it is required that either the strength of the overlying field is decreasing rapidly (Kliem \& Török 2006) or that the overlying magnetic field is sufficiently removed. The latter can occur in two ways. One way is through external reconnection (e.g. breakout), whereby the strapping field reconnects with an external ambient atmospheric field. If the orientation of the external field favours reconnection, the strapping field can be removed, leading to an ejective eruption. On the other hand, if the orientation does not favour reconnection, the strapping field is not efficiently removed and its downwards tension suppresses the eruption (e.g. Archontis \& Hood 2012; Leake et al. 2013, 2014). Another way that the tension of the overlying field can be removed is through internal reconnection. If an upwards accelerating magnetic flux rope stretches its strapping field enough, then the field lines of the strapping field can become anti-parallel and reconnect though a current sheet below the magnetic flux rope (tether-cutting reconnection; e.g. Moore \& Roumeliotis 1992). The orientation of the field lines is such that this reconnection is very efficient and accelerates the flux rope further upwards, while removing the strapping field in a runaway manner (e.g. Moore et al. 2001).

Numerical simulations focussing on the self-consistent coupling of the solar interior with the solar atmosphere have extensively studied the role of shearing, reconnection, and also ideal instabilities, in forming eruptions from bipolar regions with strong PILs (e.g. Manchester et al. 2004; An \& Magara 2013; Archontis \& Török 2008; Archontis \& Hood 2012; Leake et al. 2013, 2014; Syntelis et al. 2017, 2019a; Archontis \& Syntelis 2019). Such bipolar regions with compact PILs are usually formed when a highly twisted, but kink stable, single $\Omega$-loop flux tube partially emerges (i.e. its axis remains below or at the photosphere) from the solar interior into the solar atmosphere (e.g. Fan 2001).

Other configurations producing more complex regions with strong PILs have also been examined. For instance, the emergence of a weakly or non-twisted single $\Omega$-loop flux tubes can form a quadrupolar region, where the innermost polarities form a compact PIL (Murray et al. 2006; Archontis et al. 2013; Syntelis et al. 2015). Such simulations can mimic observations of quadrupolar regions where the two bipoles emerge simultaneously, approach each other, and form a strong PIL in between them. Reconnection at the current sheet above these PILs can lead to the formation of post-emergence low-lying flux ropes. In these examples the flux ropes remained stable.

Another approach to form quadrupolar regions is to assume a highly twisted subphotospheric flux tube that is buoyant at two locations along its length, leading to the emergence of two $\Omega$-loop segments. The segments partially emerge above the photosphere simultaneously, forming two bipoles. Again, the two innermost polarities collide forming a compact PILs similar to $\delta$-spots. It has been demonstrated that such configurations can build up shear and free energy in the corona, without however reporting eruptions and flares (Fang \& Fan 2015; Toriumi \& Takasao 2017).

Other numerical studies have shown that $\delta$-spots can also be formed when a kink unstable subphotospheric flux tube partially emerges into the atmosphere (e.g. Fan et al. 1999; Linton et al.
1999). Depending on the amount of twist of the kink-unstable flux tube, either bipolar or quadrupolar regions with compact PILs can be formed (e.g. Knizhnik et al. 2018). The shearing injected in the atmosphere is mainly associated with the photospheric vorticity, and the polarities can be flux imbalanced. In contrast, in the two $\Omega$-loops emergence of a kink stable flux tube described in the previous paragraphs, the shearing is mostly due to the horizontal motions of the polarities and the flux is mostly balanced (Takasao et al. 2015). Configurations from kink unstable flux tubes can build up free energy in the atmosphere and produce sheared structures, however, eruptions or flares have not been studied yet.

Toriumi \& Takasao (2017) examined both the emergence of a kink unstable flux tube and a double $\Omega$-loop flux tube. These authors also examined the emergence of a smaller flux tube next to a larger flux tube to mimic a parasitic polarity emerging next to a developed active region, and the simultaneous emergence of two separate flux tubes next to each other, mimicking two nearby active regions. The most compact PILs were found in the kink unstable flux tubes, followed by the double $\Omega$-loop flux tube. All cases were able to build up free energy and sheared arcade fields without however producing flares or eruptions.

Despite the significant progress in understanding the selfconsistent formation of quadrupolar $\delta$-spot regions, eruptivity and flaring are not well understood. Lee et al. (2015) investigated the emergence of a double $\Omega$-loop flux tube into a corona with an overlying field, giving rise to recurrent blowout jets driven by flux rope eruptions. However, the presence of a pre-existing coronal field significantly modifies the eruption dynamics. Thus, it is of interest to perform this experiment with a non-magnetised atmosphere and study the eruptions and flaring associated solely with the emerged quadrupolar region. We thus aim to examine further the formation of flux ropes in quadrupolar regions and the triggering mechanism of the associated eruptions.

The paper is structured as follows. Section 2 describes the numerical set-up used in the experiment. Section 3.1 discusses the initial rise and emergence of the flux tube before Sects. 3.2 and 3.3 look in more detail at the mechanisms underpinning two eruptions. Section 3.4 discusses the flaring associated with these eruptions. All the findings are summarised and discussed in Sect. 4.

\section{Numerical set-up}

\subsection{Magnetohydrodynamics equations}

We employed a three-dimensional (3D) Lagrangian-Remap code to investigate flux emergence and the following dynamics. Lare3D, developed by Arber et al. (2001), solves the timedependent, resistive and compressible magnetohydrodynamics (MHD) equations in a Cartesian geometry. The equations can be written in dimensionless form via the choice of normalisation constants, which in this case are motivated by typical photospheric values as follows: $\rho_{0}=1.67 \times 10^{-7} \mathrm{~g} \mathrm{~cm}^{-3}$, characteristic scale height $H_{0}=180 \mathrm{~km}$, and the magnetic field strength $B_{0}=300 \mathrm{G}$. This in turn constrains the remaining normalisation constants such that pressure $P_{0}=7.16 \times 10^{3} \mathrm{erg} \mathrm{cm}^{-3}$, temperature $T_{0}=623 \mathrm{~K}$, velocity $v_{0}=2.1 \mathrm{~km} \mathrm{~s}^{-1}$, and time $t_{0}=85.7 \mathrm{~s}$. The dimensionless equations become

$\frac{\partial \rho}{\partial t}+\nabla \cdot(\rho \boldsymbol{v})=0$,

$\frac{\partial(\rho \boldsymbol{v})}{\partial t}=-\nabla \cdot(\rho \boldsymbol{v} \boldsymbol{v})+(\nabla \times \boldsymbol{B}) \times \boldsymbol{B}-\nabla P+\rho g+\nabla \cdot \boldsymbol{S}$, 


$$
\begin{aligned}
& \frac{\partial(\rho \epsilon)}{\partial t}=-\nabla \cdot(\rho \boldsymbol{\epsilon})-P \nabla \cdot \boldsymbol{v}+Q_{\text {joule }}+Q_{\text {visc }} \\
& \frac{\partial B}{\partial t}=\nabla \times(\boldsymbol{v} \times \boldsymbol{B})+\eta \nabla^{2} \boldsymbol{B} \\
& \epsilon=\frac{P}{(\gamma-1) \rho}
\end{aligned}
$$

In these equations, $\rho, \boldsymbol{v}, \boldsymbol{B}, P$, and $\epsilon$ are the density, velocity vector, magnetic field vector, gas pressure, and specific internal energy, respectively. We assume an ideal gas with $\gamma=5 / 3$ and a uniform gravitational field. The quantity $S$ is the viscous stress tensor and $\eta$ is the resistivity resulting in joule dissipation of the current. The resistivity is set to be a constant background value, $\eta=0.01$, throughout the numerical domain. This high value ensures numerical stability and leads to suitable energy dissipation across the short length scales observed during the simulation. The values $Q_{\text {visc }}$ and $Q_{\text {joule }}$ are the viscous and ohmic heating, respectively.

\subsection{Model}

The experimental set-up is similar to Lee et al. (2015); the key difference is that we impose an unmagnetised corona. Figure 1 shows the initial background stratification of the atmosphere. The atmosphere is in hydrostatic equilibrium. We implemented an adiabatically stratified sub-photospheric region between $-5.4 \mathrm{Mm} \leq z<0 \mathrm{Mm}$, which is marginally stable to the convective instability. The photosphere/chromosphere is captured by an isothermal region between $0 \mathrm{Mm} \leq z \leq 1.9 \mathrm{Mm}$. Above this lies the transition region in which the temperature rises steeply between $1.9 \mathrm{Mm} \leq z \leq 2.7 \mathrm{Mm}$ and connects to the lower solar corona, which extends from $2.7 \mathrm{Mm} \leq z \leq 57.6 \mathrm{Mm}$.

A horizontal magnetic flux rope is placed within the solar interior at $z=-2.1 \mathrm{Mm}$ orientated along the $y$-direction. The axial field strength falls off in a Gaussian manner away from the centre of the flux tube, while the azimuthal field is such that the flux tube has constant twist, i.e.

$B_{y}=B_{0} \exp \left(-r^{2} / R^{2}\right)$,

$B_{\theta}=\alpha r B_{y}$.

The radius of the tube is taken to be $R=450 \mathrm{~km}$ and $r$ is the radial distance from the axis. The quantity $B_{0}$ is the field strength along the tube axis and is set to be $2.4 \mathrm{kG}$. The twist is characterised by a uniform value of $\alpha=2.2 \times 10^{-3} \mathrm{~km}^{-1}$ such that the tube is marginally stable to the kink instability. There is no pre-existing magnetic field in the corona, therefore there is no interaction between the emerging field and any ambient background field.

In order for part of the flux tube to emerge, a density deficit $\Delta \rho$ is imposed along the flux tube akin to the method used by Fan (2001). In this work, instead of having only the central part of the flux tube emerge, we initiate the buoyant emergence in two locations along the length of the flux tube, via the following profile:

$\Delta \rho=\left[p(r) / p_{\mathrm{b}}(z)-1\right] \rho_{\mathrm{b}}(z) \exp \left(-y^{2} / \lambda^{2}\right) \sin ^{2}(2 \pi y / \omega)$,

where $p$ is the gas pressure within the tube, $p_{\mathrm{b}}(z)$ is the pressure of the background atmosphere, and $\rho_{\mathrm{b}}(z)$ is the density of the background atmosphere. The equation $\lambda=3.6 \mathrm{Mm}$ is the length of the buoyant part of the tube, and $\omega=31.5 \mathrm{Mm}$ is half of the length of the flux tube.

The simulation is carried out using a uniform numerical grid made up of $420^{3}$ grid points. This represents a physical domain

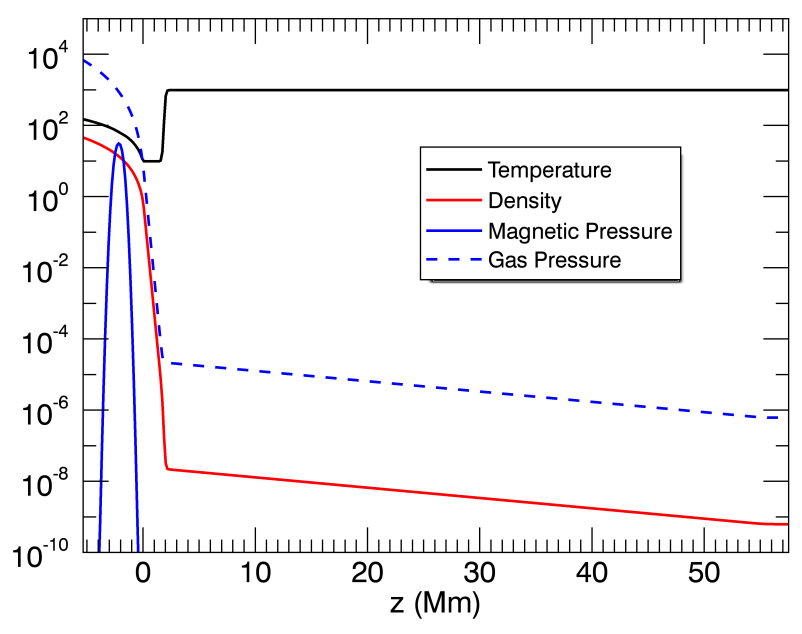

Fig. 1. Initial stratification of the solar atmosphere in our numerical experiments, in dimensionless logarithmic scale. Temperature (black), density (red), magnetic pressure (solid blue), and gas pressure (dashed blue).

of $[-31.5,31.5] \times[-31.5,31.5] \times[-5.4,57.6] \mathrm{Mm}$ in the horizontal direction perpendicular to the flux tube $(x)$, along the flux tube axis $(y)$ and the vertical direction $(z)$, respectively. Periodic boundary conditions are used in the $y$ direction whilst the upper $z$, and both the $x$ boundaries are implemented via open far-field Riemann characteristics, which allows plasma to flow out of the grid. The lower $z$ boundary is set to be a closed boundary.

\section{Results}

\subsection{Initial evolution and emergence}

We first briefly describe the overall evolution of our numerical experiment. The density deficit introduced to the flux tube means it is buoyantly unstable at two locations along its length. This causes two segments of the tube to rise, forming two $\Omega$-shaped loops (see magnetic field strength distribution, upper panel, Fig. 2). These two emerging loops continue to rise until the apex of each loop reach the photosphere $(z=0 \mathrm{Mm})$. There, both loops compress and expand horizontally, similar to a single rising $\Omega$-loop (e.g. Archontis et al. 2004). This compression increases the apex magnetic field strength (e.g. Syntelis et al. $2019 b$ ). Eventually, around $t=170.0 \mathrm{~min}$, the magnetic buoyancy instability is triggered (Acheson 1979; Archontis et al. 2004). After this, the apex field of both segments partially emerge above the photosphere and into the solar atmosphere forming two magnetic lobes (middle panel, Fig. 2).

As the flux continues to emerge, the two magnetic lobes expand both vertically and horizontally. The lobes eventually compress against each other and later reconnect to form an envelope field which encloses the newly emerging flux (e.g. lower panel, Fig. 2). The interaction of the magnetic lobes triggers dynamical behaviour throughout the system. The maximum and minimum vertical velocities in the computational domain are plotted as a function of time in Fig. 3, showing a series of plasma accelerations. The temporal correlation between the spiked upflows and downflows suggests a common source for such events. For example, the first up-flow peak at $t=172 \mathrm{~min}$ is due to the emergence of the flux tube into the corona, and the down-flow peak at a slightly later time shows the draining following the emergence. The two profound spikes of up-flows and down-flows are discussed later. 

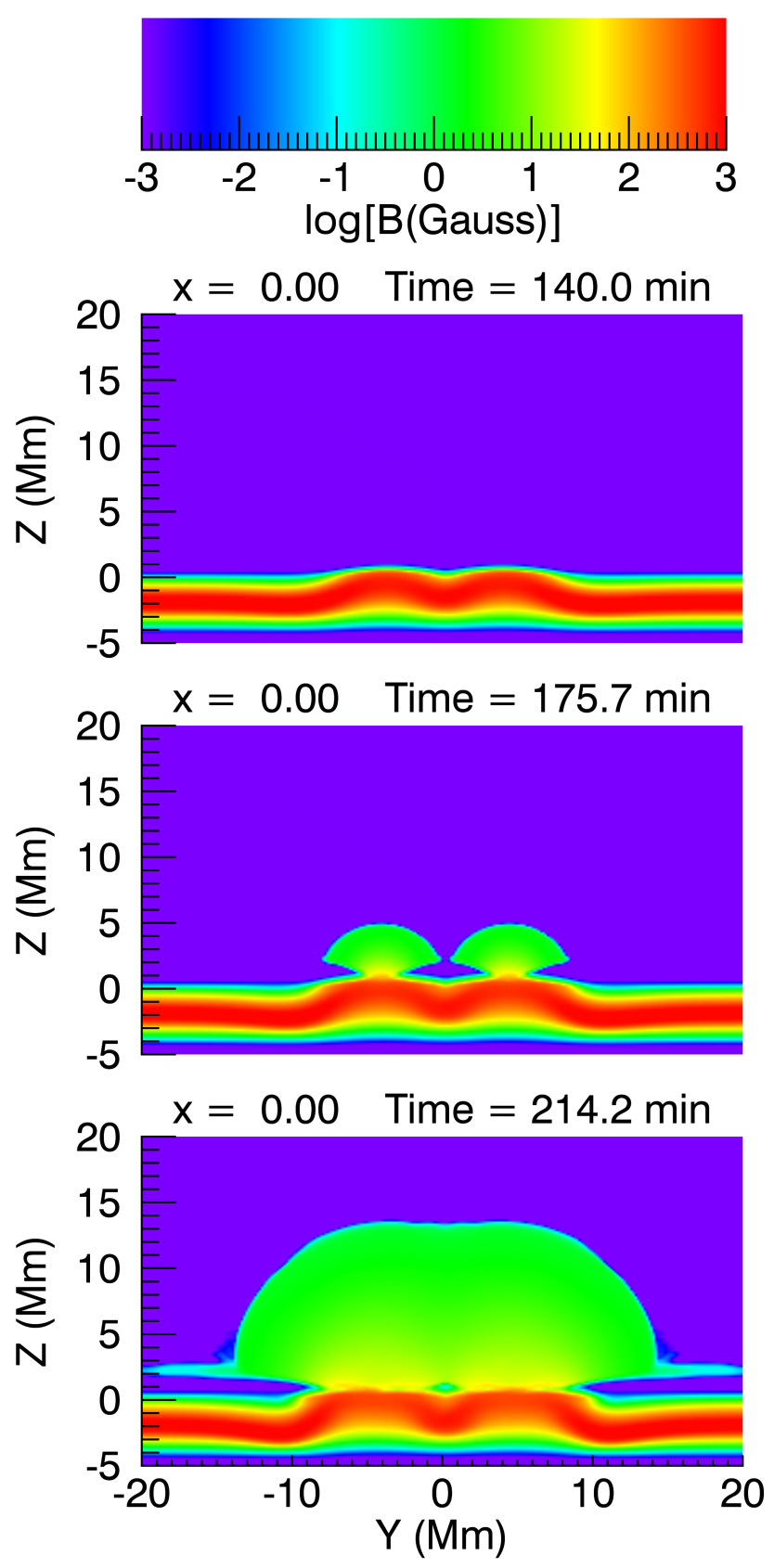

Fig. 2. Absolute magnetic field strength plotted at the central $y z$ plane $(x=0.0 \mathrm{Mm})$ intersecting the middle of the flux tube. Upper panel: emerging segments of the flux tube becoming compressed below the photosphere $(t=140.0 \mathrm{~min})$. Middle panel: initial emergence of the field above the photosphere, leading to the formation of two magnetic lobes which extend into the corona $(t=175.7 \mathrm{~min})$. Lower panel: later evolution of the two magnetic lobes, where they have further expanded, compressed against each other and eventually interacted $(t=214.2 \mathrm{~min})$

The photospheric magnetic field during the evolution of the system initially shows two well-separated bipolar regions (e.g. upper panel, Fig. 4). For clarity, we named the two bipoles as bipole 1 (right) and bipole 2 (left). Positive $B_{z}$ (red) is denoted by $P$ whilst the negative (blue) is denoted by $N$. The projected velocity field (arrows) indicates that the polarities of each bipole diverge away from each other and that converging motions develop at their internal PIL. The two bipoles move away from the location of their initial emergence, mostly moving along the $y$-axis. Eventually, the $(P 1, N 2)$ polarities come in contact,

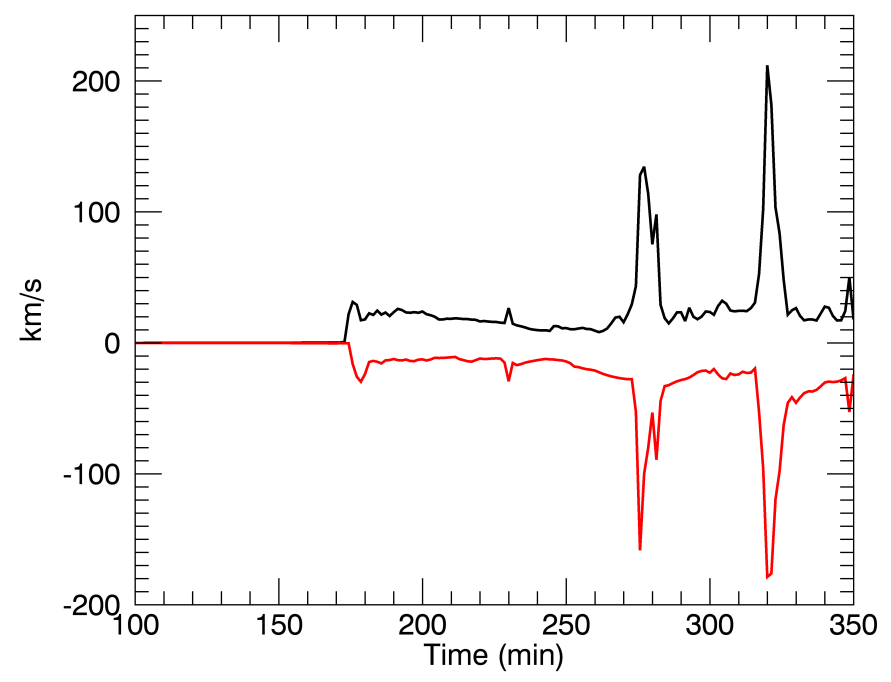

Fig. 3. Temporal evolution of maximum (black) and minimum (red) $v_{z}$ in the corona.
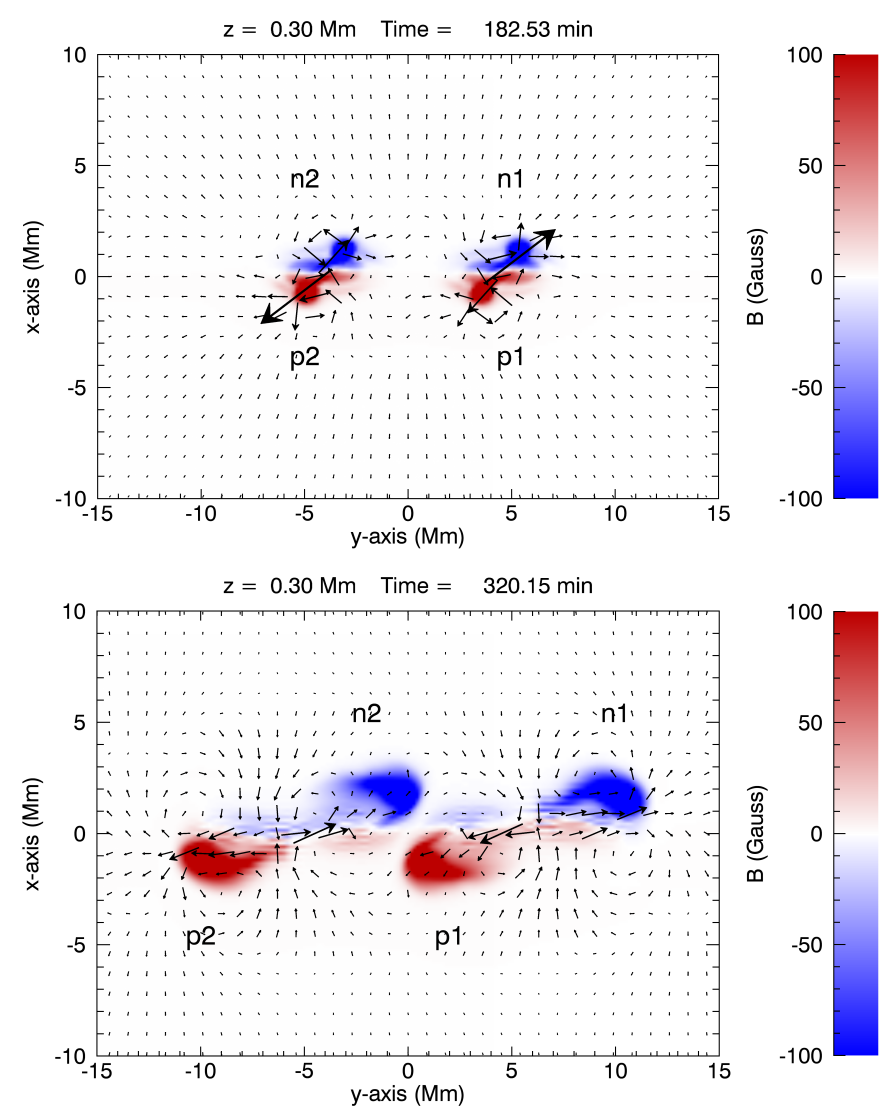

Fig. 4. Photospheric $B_{z}$ (red-blue) and the horizontal velocity (arrows). Upper panel: two bipoles shortly after the emergence $(t=182.5 \mathrm{~min})$. Lower panel: later stage of the emergence when the two bipoles have become more sheared $(t=320.0 \mathrm{~min})$

forming a new PIL in between them (e.g. lower panel, Fig. 4). Hereafter, the pair $(P 1, N 2)$ is referred to as inner polarities and the pair $(P 2, N 1)$ as outer polarities. Over time, field lines closer to the flux tube axis emerge above the photosphere; the axis of the flux tube always remains below the photosphere. These field lines inject more horizontal field and therefore more shear into the photosphere, and as a result contribute towards stressing the atmospheric field. 


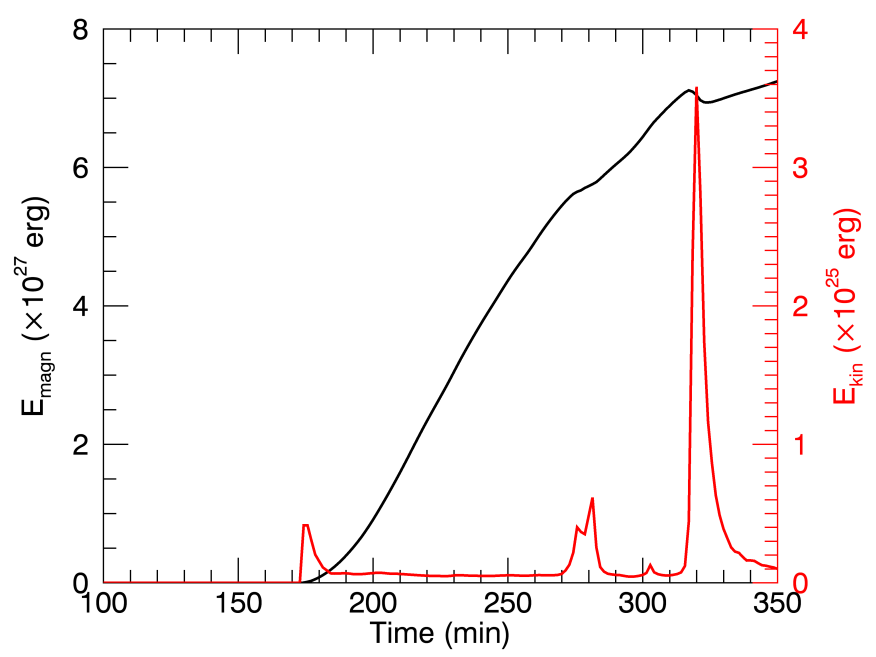

Fig. 5. Temporal evolution of total magnetic (black) and total kinetic energy (red) inside the corona.

Figure 5 shows the total coronal magnetic energy (black) and the total coronal kinetic energy (red). The magnetic energy increases throughout the simulation as flux continuously emerges into the corona. The magnetic energy starts to saturate towards the end of the simulation, signifying the end of the emergence phase. The kinetic energy exhibits two strong peaks coinciding with the profound spikes in $v_{z}$ (Fig. 3). These are associated with two flux rope eruptions. During the eruptions, the rate of magnetic energy build-up decreases as free magnetic energy is converted into kinetic energy and heat. The modulation in magnetic energy is of the order of $10^{26} \mathrm{erg}$. while the kinetic energy of the eruptions is $0.6-3.6 \times 10^{25} \mathrm{erg}$.

\subsection{First confined eruption}

We now focus on the first eruption. As described in the previous section, two magnetic lobes are formed above the emerged region (Fig. 2b). Some example lines of these two magnetic lobes, traced from around $z=13 \mathrm{Mm}$, are shown in blue in Fig. 6 $(t=274 \mathrm{~min}$, before the first eruption), connecting $(P 1, N 1)$ and $(P 2, N 2)$. It is clear that the lobes compress against each other because of their lateral expansion (panels a, b). This $3 \mathrm{D}$ expansion results in the formation of a series of current sheets at the interface between the two lobes (e.g. $|J / B|$ at the $x z$-mid-plane, Fig. 7a). A long and extended current sheet is formed higher up in the atmosphere ("upper current sheet"). It extends between 10-16 Mm and is approximately parallel to the inner PIL. Because of the 3D expansion of the lobes, compression is maximum around these heights, while the compression decreases above and below these heights. The reconnection of the lobe field lines along the upper current sheet forms field lines connecting the outer polarities $(P 2, N 1)$ (grey lines, Figs. 6c,d). These grey lines relax downwards, contain a small amount of shear and twist, and eventually adopt an almost straight configuration. Besides the grey lines, the reconnection of the lobe field lines form lines connecting the inner polarities, which are discussed in the next section.

The field lines of the two magnetic lobes located lower in the atmosphere adopt a J-like shape (pink field lines, Figs. 6a,b) because of strong shearing and rotation at their foot-points and the emergence of more horizontal field as flux emergence continues (e.g. Syntelis et al. 2017). Another current sheet, labeled the "lower current sheet", is formed at the interface between the
J-like field lines (see Fig. 7a). The reconnection between the pink lines form the field lines connecting the outer polarities (panels Figs. 6e,f) and an arcade connecting the inner polarities (not shown). These orange field lines, which are a product of reconnection between sheared lines, are twisted and form a magnetic flux rope.

We note that both the orange and grey lines are formed by the reconnection of the field lines of the two magnetic lobes. However, because the reconnection occurs at different heights, the blue and pink lines that reconnect have a different amount of shear and a different orientation. Therefore, the resulting grey and orange lines also have a different amount of shear and twist and a different orientation (e.g. relative angle between grey and orange lines in Figs. 6d,f). The collapsing grey field lines press down on top of the flux rope generating a "middle current sheet" (Figs. 7a and 6a).

The flux rope core is associated with cooler temperatures, higher densities, and a dominant axial magnetic field component. We therefore identify the axis of the flux rope by examining the temperature, density, and axial magnetic field across 2D vertical planes perpendicular to the flux rope (similar to Syntelis et al. 2017). The height-time profile of the flux rope is shown in Fig. 8 in black. The height-time profile of the flux rope exhibits three phases: (i) a slow rise phase between 271.5 and $275.6 \mathrm{~min}$, (ii) a temporary inhibition of the slow rise between 275.6 and $278.5 \mathrm{~min}$, and (iii) an eruptive phase leading to a confinement between 278.5 and $284.2 \mathrm{~min}$

These rise phases are closely related to reconnection occurring at the current sheets of the system. Thus, we follow the temporal evolution of the maximum $J /(c B)$ at the lower, middle, and upper current sheets (Fig. 9). During the first phase (until first vertical line), the flux rope rises because of magnetic pressure gradient force. As the flux rope pushes upwards against the overlying grey field lines, the middle current sheet is enhanced.

During the second phase, between $t=275.6-278.5 \mathrm{~min}$, the upper current sheet exhibits a sudden bust of reconnection delineated by the red line (Figs. 7b and 9). The long and thin upper current sheet becomes fragmented, forming two regions of increased current density and triggering fast reconnection flows. This causes the first of the two peaks associated with the $v_{z}$ and kinetic energy increase between $t=275.6-278.5 \mathrm{~min}$ in Fig. 3 and Fig. 5, respectively. This fragmentation is suggestive of a tearing instability. This sudden reconnection results in the formation of more grey field lines overlying the flux rope, and also temporarily increases the axial flux above the rope between $t=275.6-278.5 \mathrm{~min}$. These newly formed grey lines retract downwards and inhibit the rise of the flux rope between $t=275.6-278.5 \mathrm{~min}$. The average downwards tension above the middle current sheet (Fig. 8) before $t=275.6 \mathrm{~min}$ is decreasing gradually due to the expansion of the magnetised volume. Between $t=275.6-278.5 \mathrm{~min}$, however, this gradual decrease of the tension stops owing to the retracting grey lines, indicating how these lines inhibit the rise of the flux rope.

As the flux rope push upwards and the grey field lines push downwards, the middle current sheet becomes enhanced. In addition to that, between 271.5 and $278.5 \mathrm{~min}$, as the flux rope becomes more sheared and moves upwards, the average relative angle between the apex of the flux rope and the grey field lines above the middle current sheet increases (Fig. 10); the maximum relative angle has values around $90^{\circ}$. Eventually, the reconnection between the field lines of the apex of the flux rope and the grey field lines above the middle current sheet becomes more efficient, marking the beginning of the eruption of the first flux rope at $278.5 \mathrm{~min}$ (second of the two peaks associated with the 

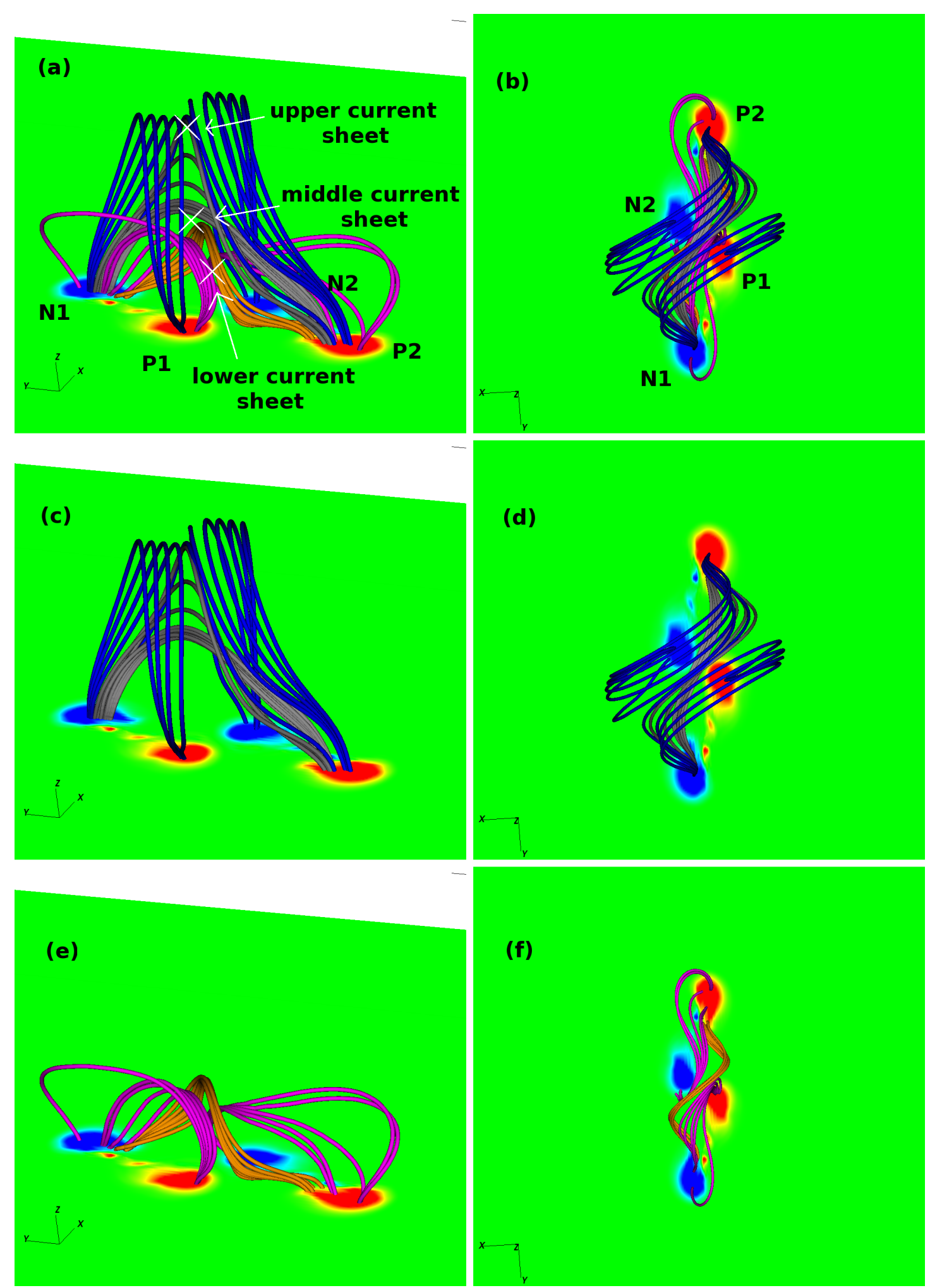

(f)

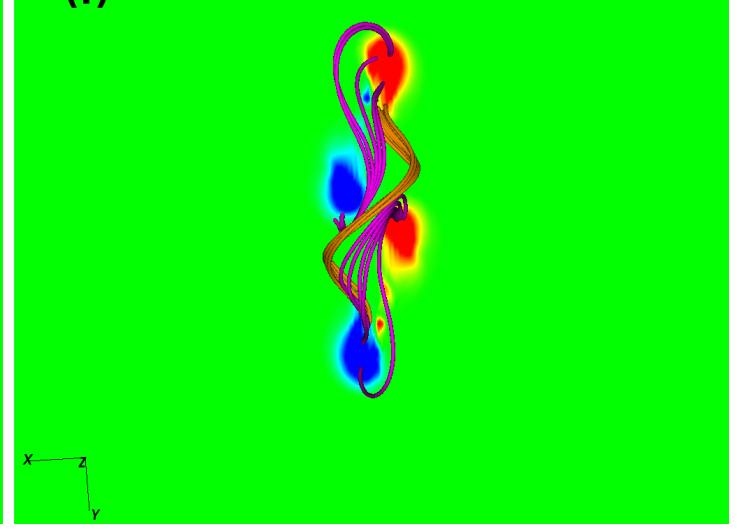

Fig. 6. Overall magnetic field line topology before the first eruption $t=274 \mathrm{~min}((a)$ side view, $(b)$ top view). The white crosses indicate the locations at which the current sheets are formed. Panels $c$ and $d$ : focus on the upper part of the field line system. Blue field lines are part of the magnetic field lobes traced around $z=13 \mathrm{Mm}$. The grey field lines result from the reconnection between blue field lines. Panels e and $f$ : lower part of the magnetic field line system. Pink field lines are lower-lying sheared field lines of the magnetic lobes. The orange field lines result from the reconnection between pink field lines. The blue-red patches at the photospheric plane show the negative-positive $B_{z}$ component of the magnetic field saturated at $\pm 300 \mathrm{G}$.

$v_{z}$ and kinetic energy increase between $t=275.6-278.5 \mathrm{~min}$ in Fig. 3 and Fig. 5, respectively). The reconnection intensifies at the middle current sheet, reducing the magnetic tension of the field above it ( $t=280 \mathrm{~min}$, red line, Fig. 8). This process is visualised in Figs. 11a,b where the flux rope (orange) and the overlying field (grey) reconnect through the middle current sheet 

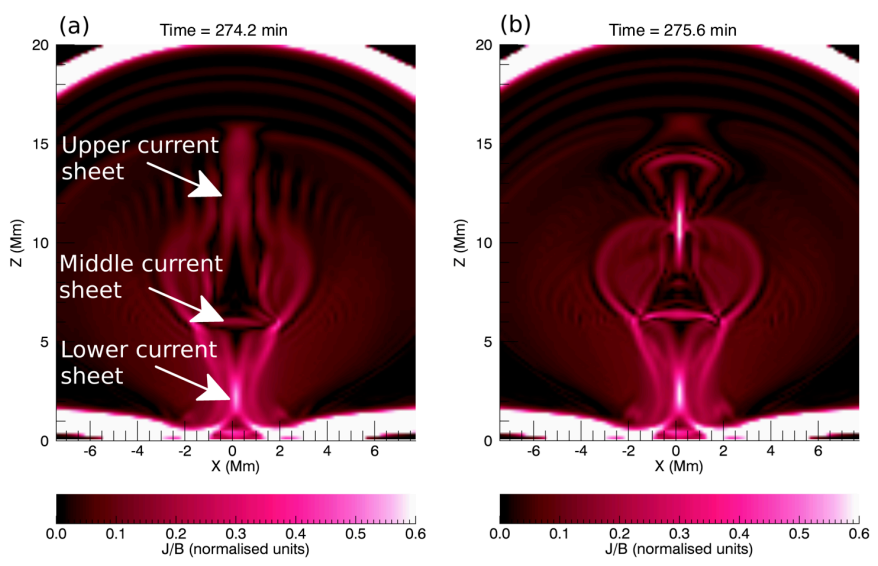

Fig. 7. $|J / B|$ at the $x z$-midplane of the numerical domain, showing the lower, middle, and upper current sheets at (a) $t=274.2 \mathrm{~min}$ and (b) $t=275.6 \mathrm{~min}$.

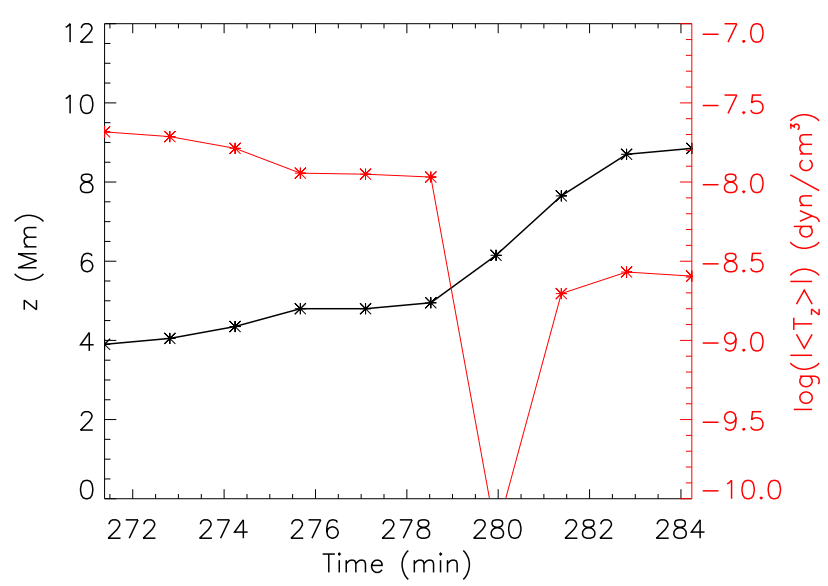

Fig. 8. Height-time profile for the first flux rope (black line) and the average $\log \left(\left|T_{z}\right|\right)$ above the middle current sheet (red line).

(purple isosurface) to form the blue lines. These newly formed blue lines have less downwards tension and are shifted away from the flux rope apex, aiding the eruption. As the flux rope erupts, the lower current sheet is also enhanced.

Eventually, most of the field of the flux rope reconnects with the field above it and the eruption stops. The resulting field after the eruption contains a structure (orange, Figs. 12a,b), consisting of some remaining flux rope lines, some grey lines, and some of the lines formed from the reconnection between the two.

\subsection{Second confined eruption}

We now focus on the second eruption. The pre-eruptive configuration is visualised in Figs. 13a,b. We again identify lowlying J-shaped field lines (pink), which are now more sheared. These lines reconnect, forming a second twisted flux rope core (orange). Above the flux rope, field lines connect the outer polarities in a similar manner to the first eruption (grey lines). These grey lines consist of the remnants of the first eruption and the continued retraction of reconnected lines at the upper current sheet.

We trace the height-time profile of the second flux rope in Fig. 14 (black) and over-plot the average tension above the flux rope (red). The initial slow rise of the flux rope $(t=308.5-314.2 \mathrm{~min})$ before the first vertical line progresses

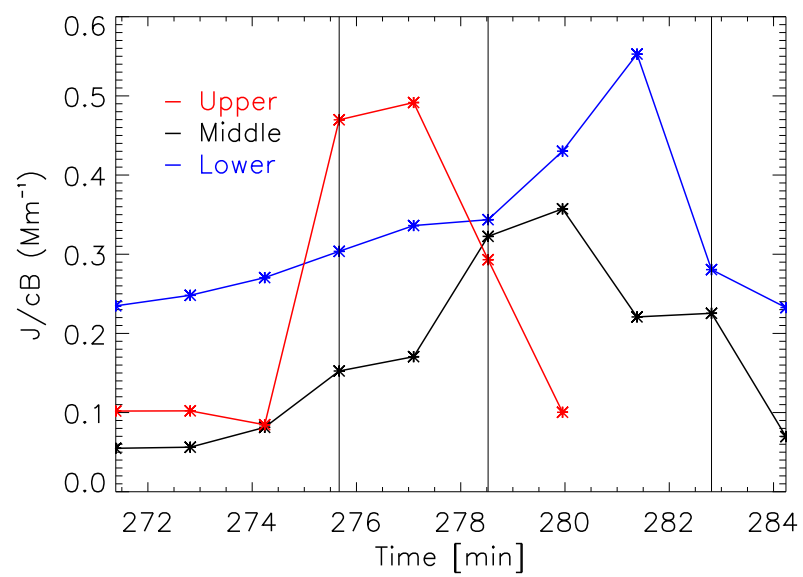

Fig. 9. Temporal evolution of $J /(c B)$ at the lower current sheet (blue), middle current sheet (black), and upper current sheet (red). The vertical lines delimit the characteristic phases of the flux rope evolution.

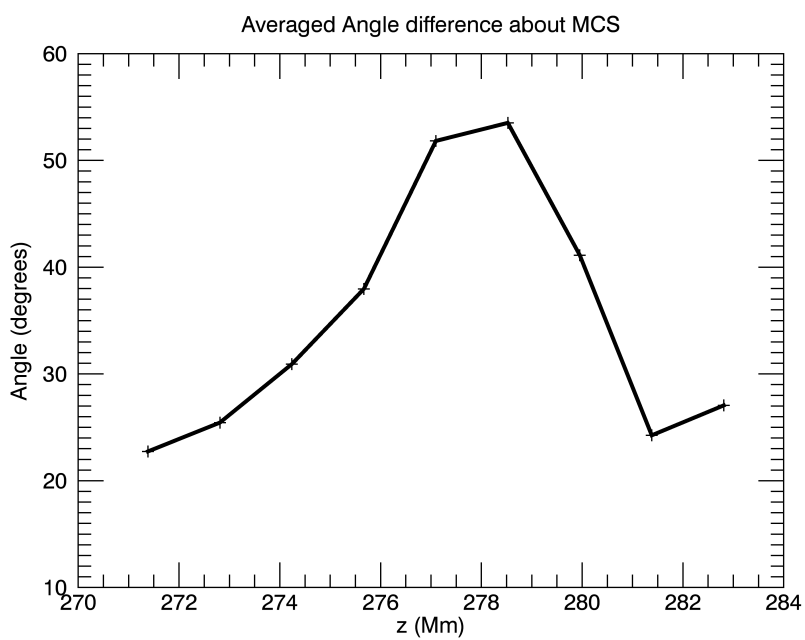

Fig. 10. Average angle difference between the field lines of the first flux rope (orange lines, Fig. 6f) and the field lines directly above it (grey lines, Fig. 6d). The average difference angle is measured between lines of the apex of the flux rope and the field lines above the middle current sheet.

towards a non-linear phase ( $t=314.2-317.1 \mathrm{~min})$ between the first and second vertical lines. This is then followed by a rapid eruption, triggered at $t=318.5 \mathrm{~min}$ after second vertical line. Eventually, the flux rope eruption becomes confined after the third vertical line.

Similar to the first eruption, the reconnection between the flux rope apex field lines and the middle current sheet is enhanced over time, reducing the tension of the overlying field (red line, first vertical line Fig. 14). This release of tension makes the flux rope move upwards, enhancing the reconnection at both the lower and middle current sheets; the bidirectional outflows (upper panel, Fig. 15) from both the lower current sheet (around $z=3 \mathrm{Mm}$ ) and the middle current sheet (around $z=7 \mathrm{Mm}$ ) start to increase from $t=314.2 \mathrm{~min}$.

As the flux rope accelerates upwards $(t=314.2-317.1 \mathrm{~min})$, more magnetic field flows into the lower current sheet and reconnects. Because this field (e.g. pink lines, Fig. 13a) is more sheared than in the first eruption, this reconnection now produces new lines that have higher upwards tension. As a result, from $t=314.2-317.1 \mathrm{~min}$ the ratio of the upwards magnetic tension over the upwards magnetic pressure along height (black, 

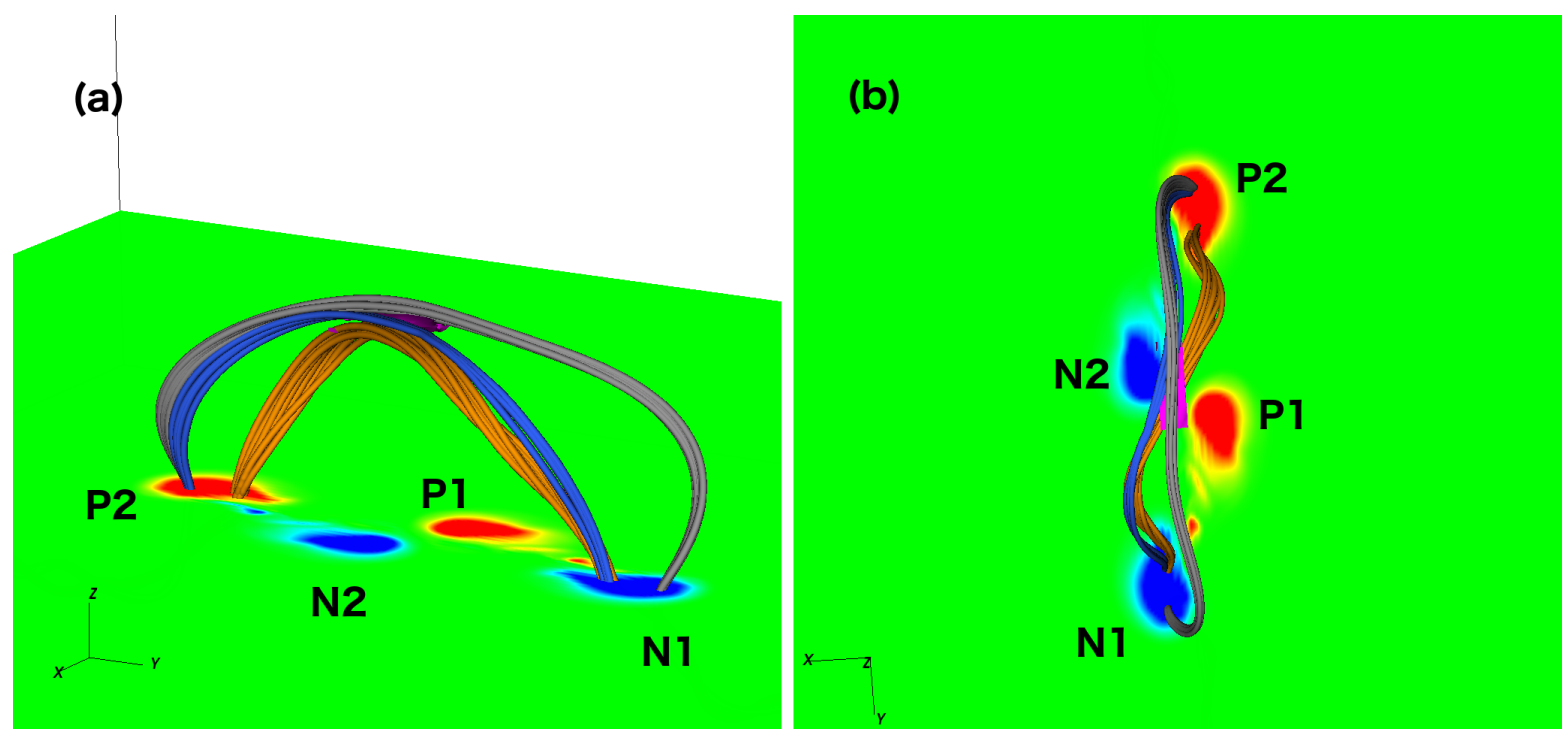

Fig. 11. Side $(a)$ and top $(b)$ view of the magnetic field line topology at $t=281.4$ min, showing the reconnection of field lines between the flux rope and the overlying field. Purple isosurface shows the middle current sheet.

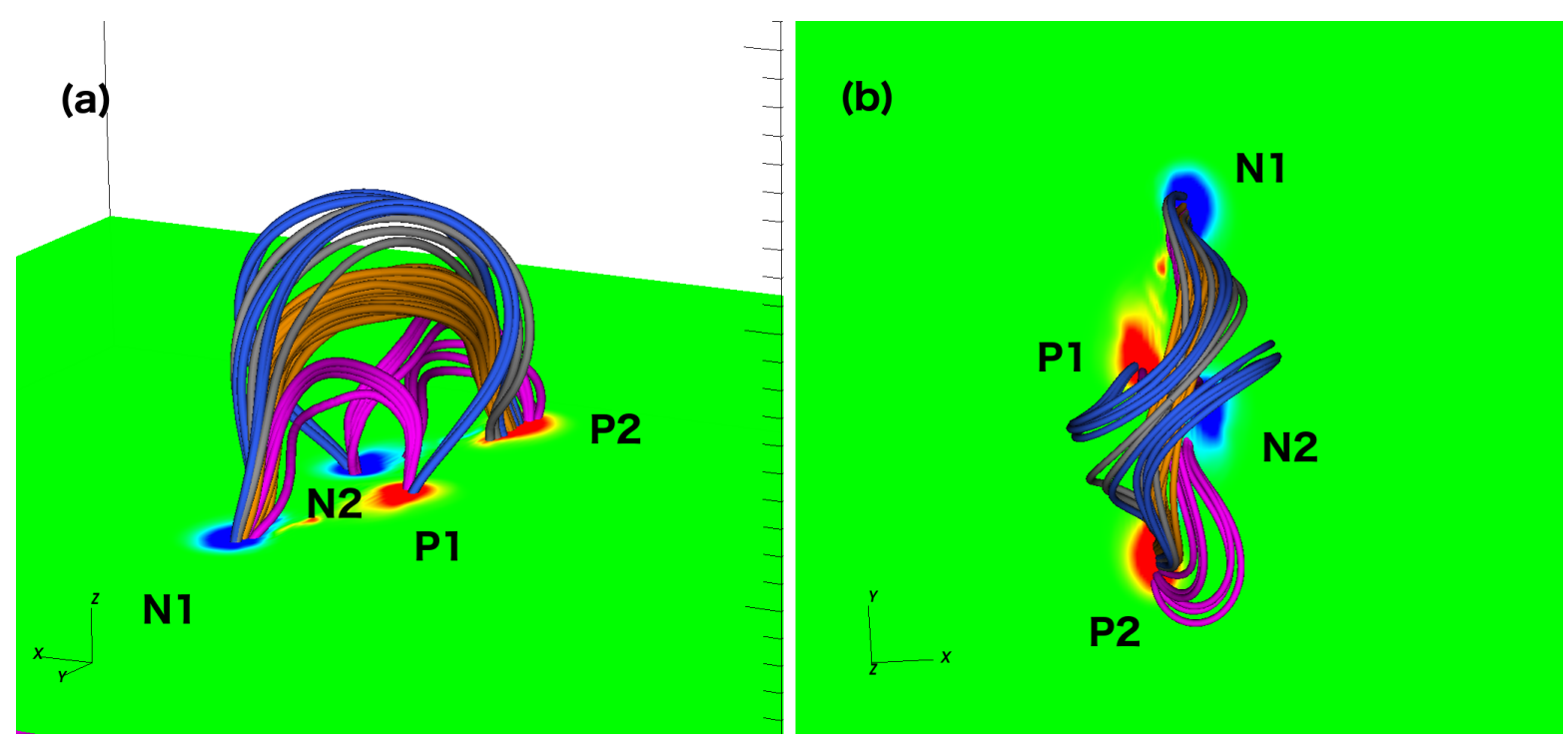

Fig. 12. Side ( $a$ ) and top $(b)$ view of the magnetic field line topology at $t=283 \mathrm{~min}$, after the first eruption.

red, and blue solid lines; Fig. 16) shows an increase by a factor of 12 below the flux rope centre indicated by the black, red, and blue vertical dashed lines. This upwards tension release from the lower current sheet drives the flux rope upwards and enhances reconnection at the middle current sheet. The reconnection rate at the middle current sheet, between field lines of the flux rope apex and field lines above the current sheet, increases over time in a runaway manner.

Eventually, the reconnection at the middle current sheet becomes very enhanced $(t=317.1 \mathrm{~min}$, joule heating peak at the location of the black vertical dashed line, Fig. 17). This becomes a rapid release of the tension of the overlying the flux rope (red line, second vertical line, Fig. 14), triggering the eruption. The corresponding outflows from the lower current sheet increase from 10 to $150 \mathrm{~km} \mathrm{~s}^{-1}$ (lower panel, Fig. 15), forming a strong reconnection jet that is ejected from the lower (now flare) current sheet. The field lines consisting of the fast reconnection reconnection outflow have a U-loop shape, further enhancing the upwards tension release below the flux rope (red lines, Figs. 13c,d) and thus further pushing the flux rope upwards. These twisted red field lines wind around the orange flux rope core, enhancing the magnetic flux of the erupting flux rope.

However, similar to the first eruption, the sudden rise of the flux rope does not enhance the middle current sheet sufficiently to remove all the overlying tension. Indeed, the Joule dissipation at the middle current sheet does eventually drop off (blue vertical dashed line, Fig. 17) and consequently, the tension of the overlying field reduces at a slower rate (red line, Fig. 14). In addition, the flux rope does not stretch the overlying field to the extent where tether-cutting reconnection is triggered. Eventually, the eruption is halted by the tension of the overlying field (after third vertical line, Fig. 14) and the flux rope eruption becomes confined by the magnetic envelope (green and grey lines, Figs. 13e,f)

Figures 13e,f show the field lines of the system after the eruption becomes confined. The flux rope (orange and red) has increased in size and flux and is now more twisted. Below the 


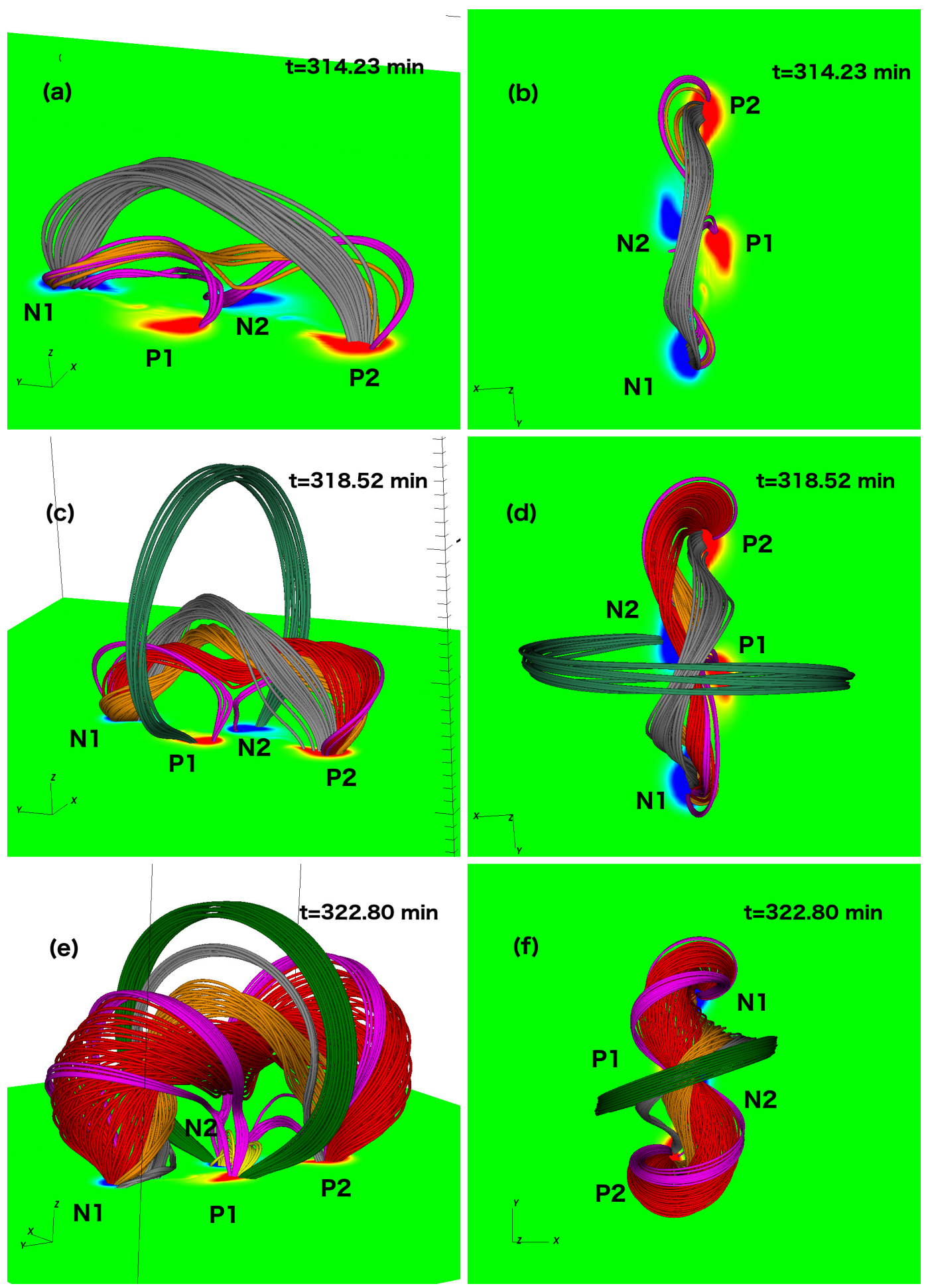

Fig. 13. Side $(a)$ and top $(b)$ view of the magnetic field line topology of the second flux rope before its eruption $(t=314 \mathrm{~min})$. The field lines are similar to the previous eruption and are coloured as in Fig. 6. Panels $c$ and $d$ : flux rope during the eruption at $t=318.5$ min. Green field lines result from the reconnection of field lines similar to the blue field lines of Fig. 12a, and act as a strapping field to the eruption. Red field lines are formed by the reconnection of pink lines during the eruption of the flux rope core (orange). Panels $e$ and $f$ : magnetic field line topology at $t=322.8 \mathrm{~min}$, when the eruption has stopped and the flux rope has been confined. The yellow field lines connecting the inner polarities $(P 1, N 2)$ are the post-reconnection arcade loops.

flux rope, a post-reconnection flare arcade connecting the inner polarities has been formed (yellow). Some of the J-like field lines (pink) have been stretched during the eruption, without reconnecting, and provide tension holding down the flanks of the flux rope. Above this, the green and grey overlying field lines act as a strapping field. It is worth mentioning that the green and grey field lines are the two products of the reconnection higher up in the atmosphere between the two original magnetic lobes (blue, Fig. 6). Both of these field line systems should develop naturally in any quardupolar region and can suppress an eruptive flux rope. 


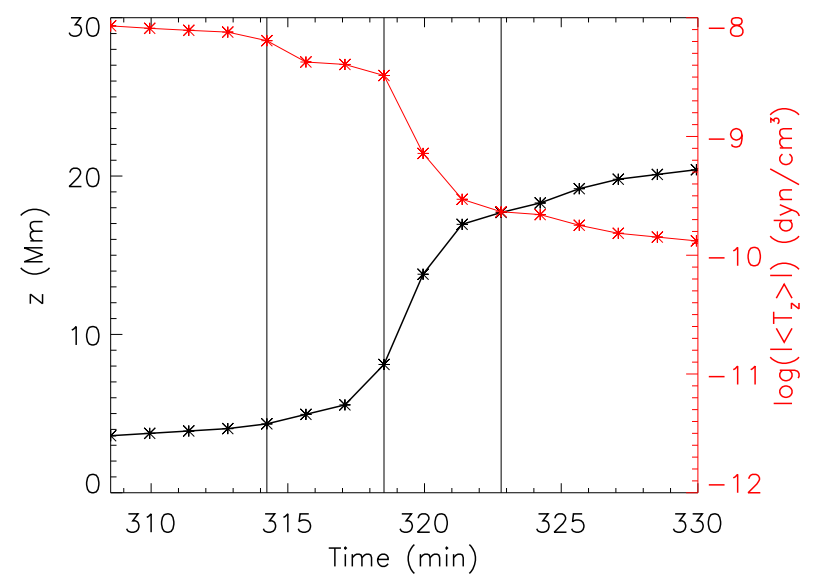

Fig. 14. Black line: height-time profile of the second flux rope. The vertical lines indicate the beginning of the gradual acceleration phase, the beginning of the eruptive phase, and the confinement phase. Red line: temporal evolution of the average absolute magnetic tension in a cross-sectional cut area above the flux rope.
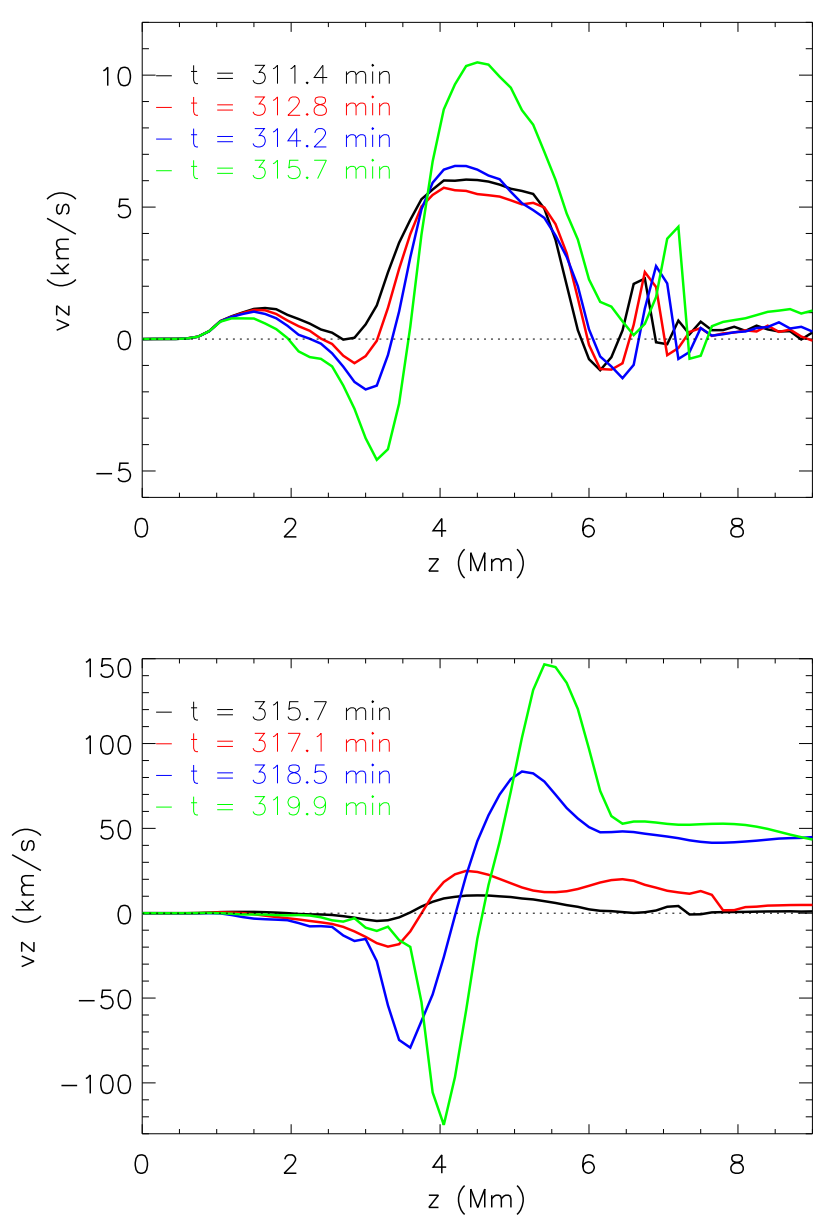

Fig. 15. At different times, $v_{z}$ along $(0,0, z)$ denoted by colour. Upper panel: earlier times; lower panel: continuation at later times. We see strong bidirectional jets develop as reconnection drives an outflow from the lower current sheet.

We note that the green lines form a third magnetic "lobe", along with the two associated with the two emerged bipoles (blue lobes of Fig. 6). Our analysis show that this structure plays a critical role in the dynamics associated with quadrupolar regions.

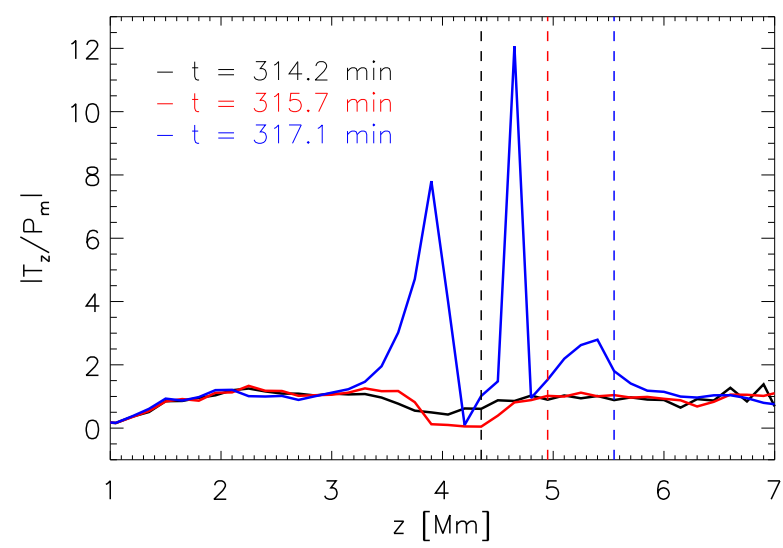

Fig. 16. Ratio of the absolute value of the vertical component of the magnetic tension force over the magnetic pressure force along $(0,0, z)$ at different times. The vertical dashed lines indicate the location of the flux rope centre at these times.

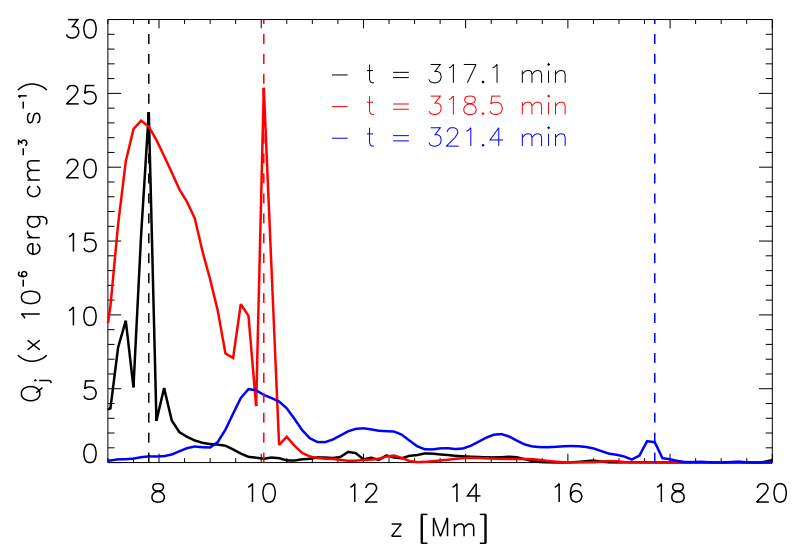

Fig. 17. Joule heating $\left(Q_{j}\right)$ along $(0,0, z)$ at different times. The vertical dashed lines indicate the location of the middle current sheet above the flux rope at these times.

\subsection{Pseudo-emission and flaring}

We generated an emission proxy (EP) to further examine the two eruptions. To do so, we integrated the density of plasma, within a specified temperature interval, over a line of sight:

$\mathrm{EP}=\int g(T) \rho^{2} \mathrm{~d} s$

where $g(T)$ is 1 inside a selected temperature interval and 0 outside it. We adopted a temperature interval of 30000-300 $000 \mathrm{~K}$, which is typical for cooler plasma in the chromospheric and transition region range, and a temperature interval of $0.6-2 \mathrm{MK}$, which is typical for hotter coronal plasma.

The emission proxies are generated during the first (left column) and second (right column) eruption, for the cooler (Fig. 18) and hotter (Fig. 19) temperature intervals, respectively. The upper, middle, and lower panels are integrated over the $x, y$, and $z$ line of sights, respectively. Contours of the $B_{z}$ field component at the photosphere were over-plotted for the $z$ line of sight to visualise the photospheric field configuration.

The flux ropes of both eruptions can be seen in the cooler temperature interval (Figs. 18a,b,c,d). In fact, these cool structures are the filament channels associated with the eruptions. The flux rope of the second and more energetic eruption appears to adopt the shape of the number eight in the hotter temperature intervals 


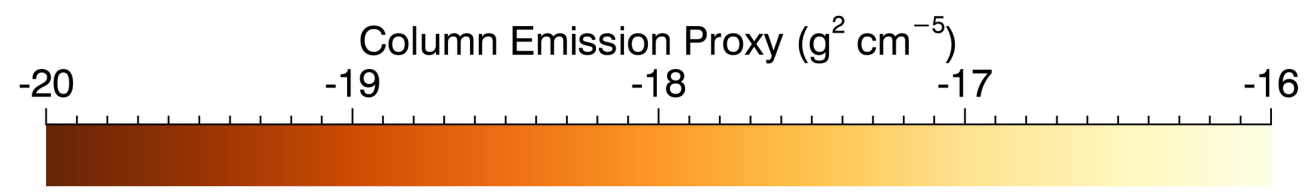

Time: $281.48 \mathrm{~min}$
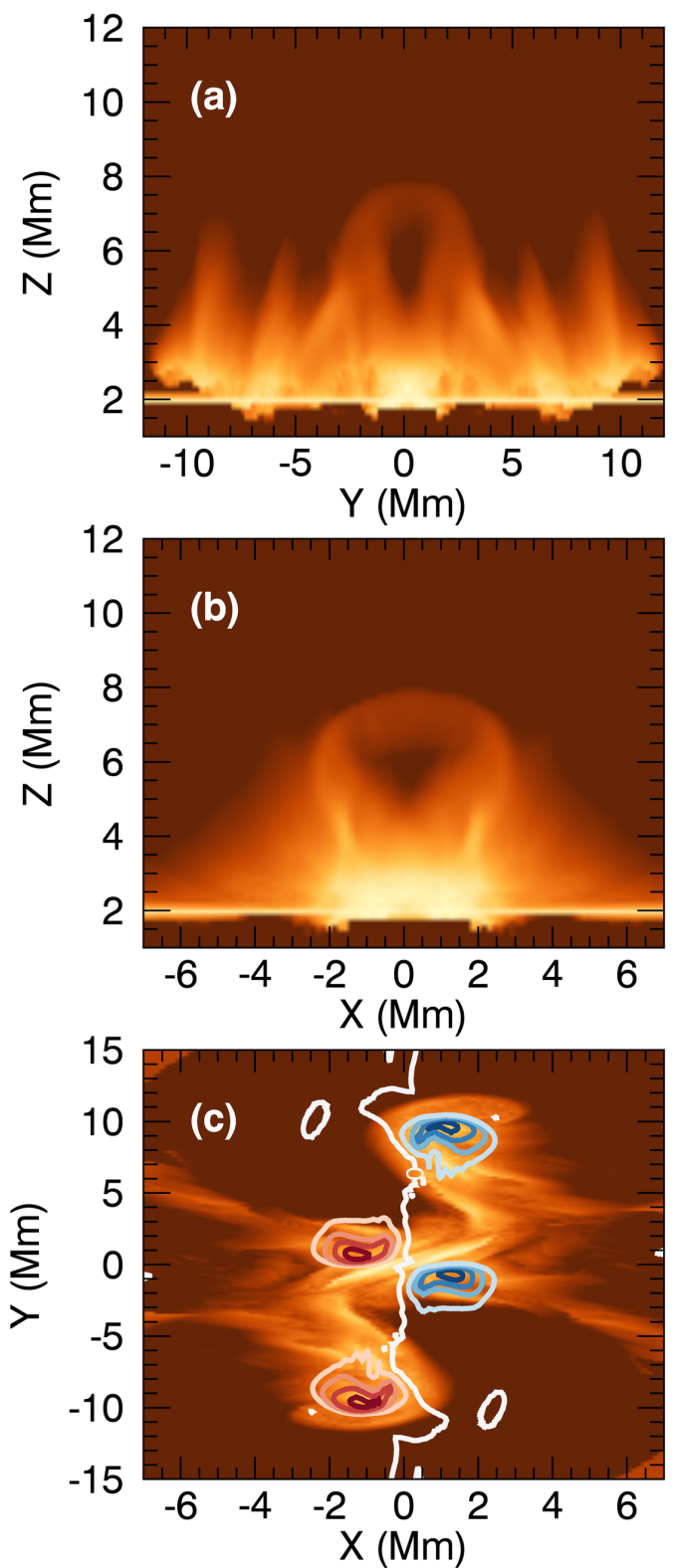

Time: $320.06 \min$
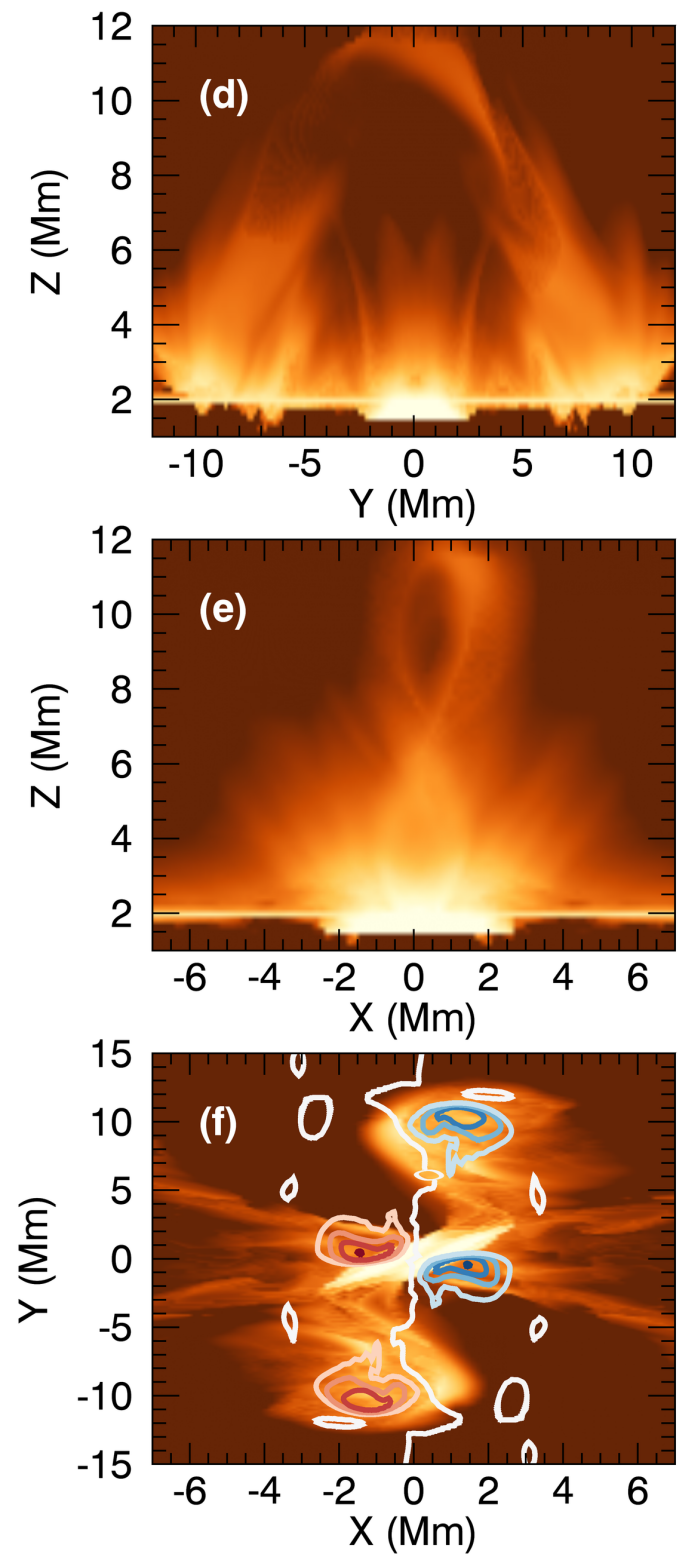

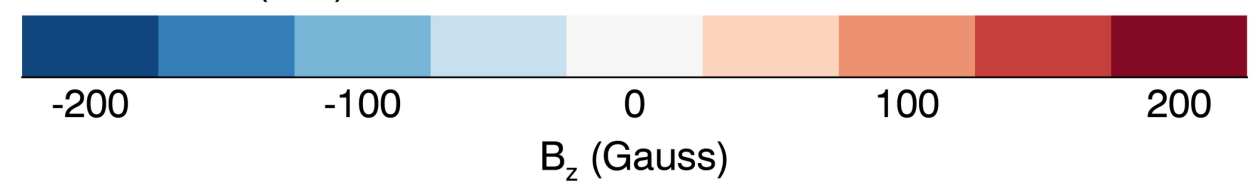

Fig. 18. Emission proxy images for the cooler temperature range $(30000-300000 \mathrm{~K})$ for the first eruption at $t=285.8$ min $($ left column) and the second eruption at $t=320.1$ min (right column). The upper, middle, and lower panels correspond to integrating over the $x$, $y$, and $z$ lines of sight, respectively. The $B_{z}$ magnetic field contours at the photosphere are overlaid in the lower panel.

(Figs. 19d,e) with a hot core between $z=7.5-9.5 \mathrm{Mm}$ (Fig. 19e). The flux rope of the first eruption is not visible in the hotter temperature intervals, as the eruption is weaker. The hot structure in Fig. $19 \mathrm{~b}$ is the current sheet developing during the eruption.

An important difference between the two eruptions is found at the location of the post-reconnection arcade. During both eruptions, arcade-like loops form below the erupting flux rope similar to the yellow lines in Fig. 13e. In the cooler temperature interval, the arcade is visible for both eruptions and is located below the lower current sheet. This is the bright band along the PIL, separating the inner polarities (e.g. Figs. 18c,f). However, in the hotter temperature interval, the arcade appears only in the 


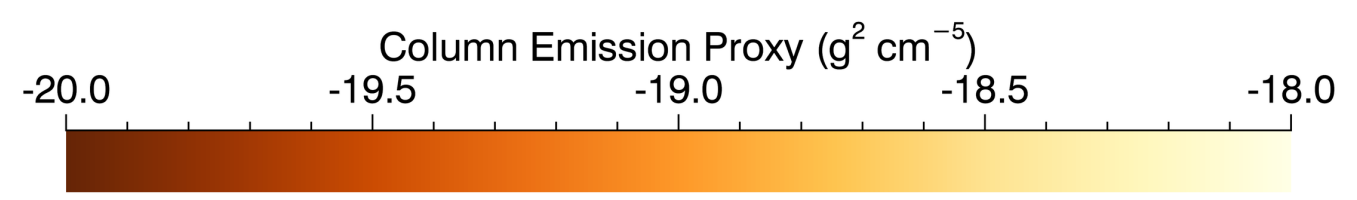

Time: $281.48 \mathrm{~min}$
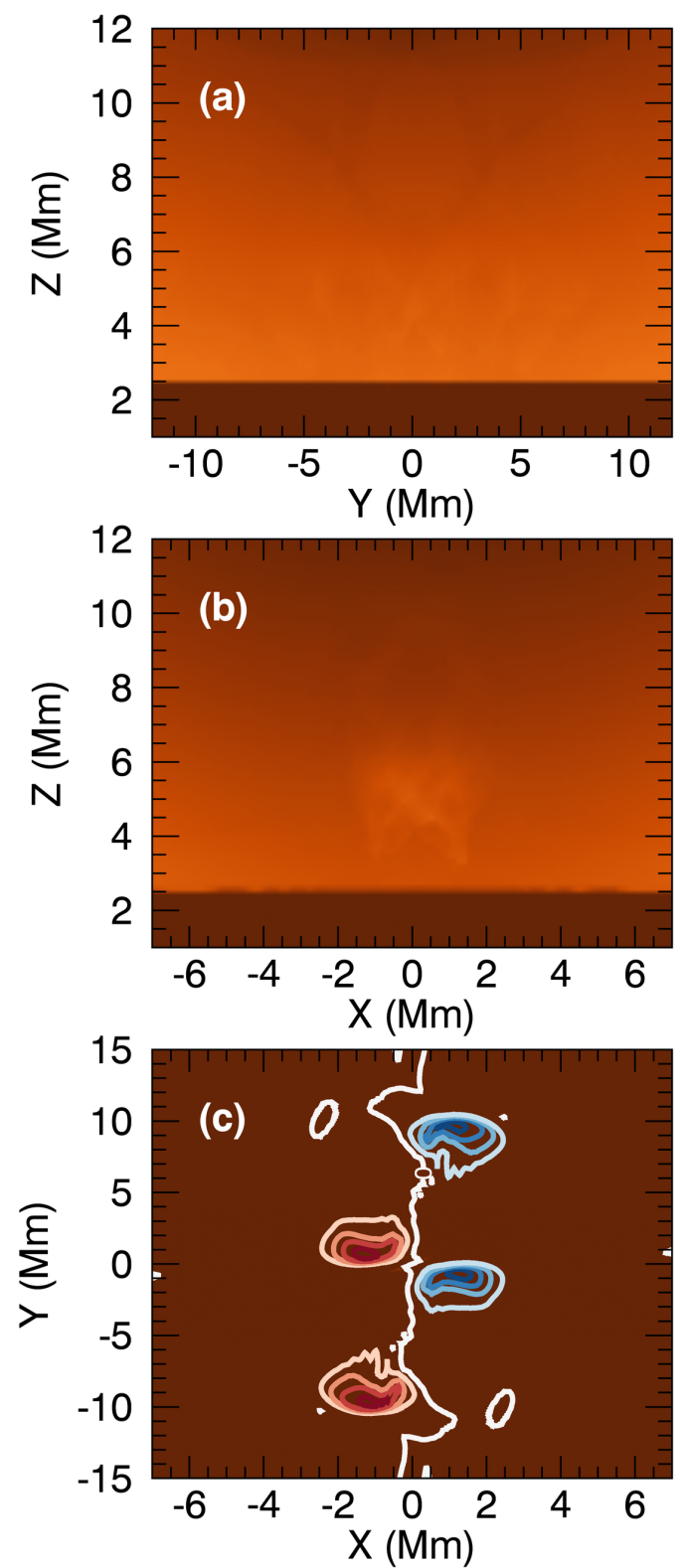

Time: $320.06 \mathrm{~min}$
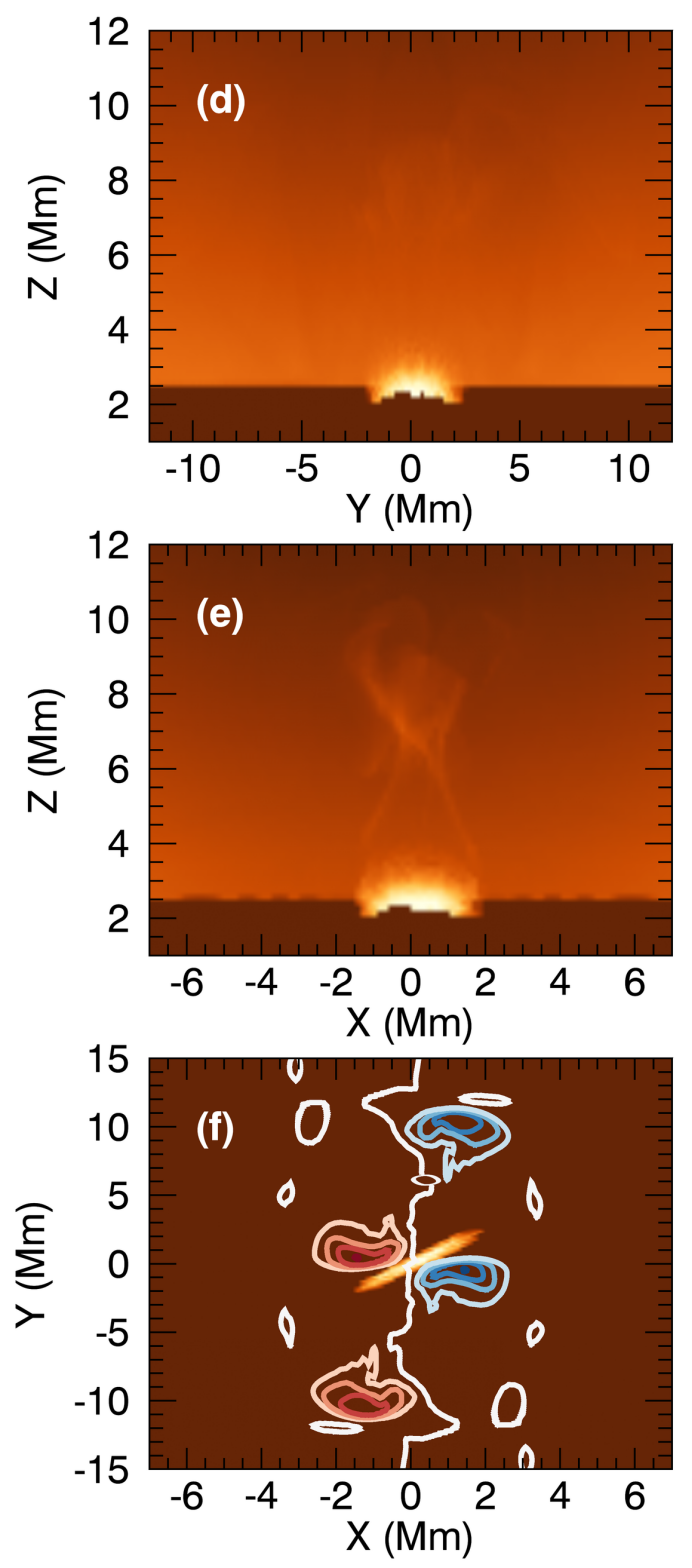

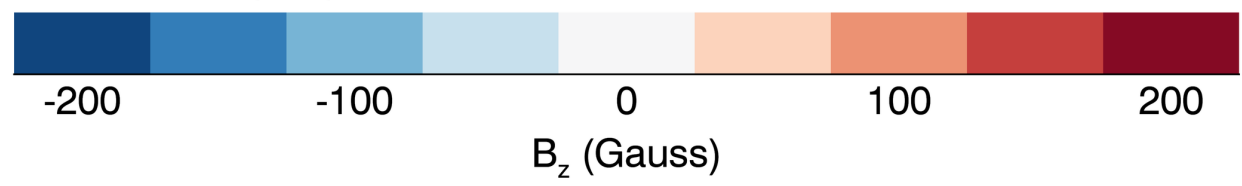

Fig. 19. Same as Fig. 18 but for the hotter temperature range (0.6-2 MK).

second eruption (Fig. 19). Therefore the arcade flares only in the second eruption and not the first.

The reason for this is simply that the second eruption is more energetic. The flux rope associated with the second eruption accelerates more, becomes bigger in size and flux, and the eventual eruption has six times more kinetic energy. There are various reasons for this. During the eruptions, the magnetic energy (Fig. 5) and photospheric unsigned flux is still increasing. This suggests there is more magnetic free energy available for release at the second eruption. Also, since the magnetic lobes continue to expand with time, the topmost field strength decreases. Thus, reconnection at the middle current sheet allows the flux rope to penetrate higher into the atmosphere (where the tension force is less) allowing the eruption to develop more before it eventually becomes suppressed. This further upwards rise allows more time for reconnection through the lower current sheet during 
the eruptive phase. The reconnection at the lower current sheet during the eruptions is further affected by other parameters. The continued shearing and compression of the inner polarities, along with the rotation of the polarities, has brought them closer together by the onset of the second eruption. This causes field lines to interact with angles more favourable to efficient reconnection. Also, the polarities of each of the bipoles have rotated more, making the field lines more curved and thus increasing the angle of reconnection further. This results in faster jets and increased upwards tension from the U-shaped field lines. As discussed in Sect. 3.3, this process assists the eruption extending the duration of the reconnection through the lower current sheet, and increasing the efficiency of the reconnection. Similarly, this results in strong downwards release of tension from $\cap$-shaped lines, which carry high temperature plasma to the postreconnection arcade, but also compress the arcade and heat the plasma locally via shocks.

\section{Summary and discussion}

In this paper, we have presented results from a 3D flux emergence simulation of a quadrupolar region and analysed the subsequent eruptive dynamics. We placed a magnetic flux tube in the solar interior and triggered its emergence at two locations along its length to form a pair of bipoles at the photosphere. During the evolution of the system, the two initially separated bipoles converge, forming a $\delta$-shaped region between the two inner polarities of the quadrupolar region. Inside the atmosphere, the field expands outwards forming two magnetic lobes that eventually interact through a series of current sheets. At the PIL between the inner polarities, sheared field lines reconnect and form two flux ropes that erupt in a confined manner.

The two successive eruptions have distinct rise phases controlled by reconnection at the lower (below the flux rope), middle (above the flux rope) and upper current sheets (further above above the flux rope). In both cases, slow low-lying tether cutting reconnection through the lower current sheet gradually builds up a flux rope, which moves upwards by the magnetic pressure gradient. At the same time, the reconnection between the two lobes higher in the atmosphere (at the upper current sheet) forms field lines that retract down pushing against the flux rope, creating the middle current sheet between them. The reconnection rate between the rising flux rope and the retracted field changes over time as the relative angle of the field lines of the two systems increase and as the flux ropes moves upwards because of the emergence, shearing, and rotation of the photospheric field.

The triggering of both eruptions occurs when the reconnection between the rising flux rope and the field directly above it becomes efficient. Our simulation assumes a non-magnetised atmosphere, therefore, there is no external reconnection between the quadrupolar system and any pre-existing atmospheric field. Despite the lack of any external field, the triggering mechanism of the eruptions is similar to the process of the external reconnection, as both flux ropes erupt when the tension of the dynamically formed, overlying field is reduced. It is well known that the relative angle between two magnetic field systems can produce or suppress an eruption (e.g. Archontis \& Hood 2012). It is therefore very reasonable that in this work we find the triggering of both eruptions to be affected again by the relative angle of the two interacting systems.

After the initial acceleration phase, both eruptions become confined by the overlying field (green and grey lines, Fig. 13e) and the previously emerged stretched lines (pink lines, Fig. 13e). The overlying strapping field is the result of the reconnection between the two magnetic lobes that initially emerged. However, in principle, if the eruptions were stronger the strapping field could have been fully removed leading to an ejective eruption. Therefore, our results indicate that depending on the internal structure of the field of a quadrupolar region, a flux rope can become eruptive even without any reconnection between the field of the quadrupolar region and an ambient external field.

During the formation of the first flux rope, less magnetic energy is available. Also, the field that reconnects to form the flux rope is less sheared. This results in a smaller flux rope and an overall weaker eruption. This first eruptive flux rope almost fully reconnects with the field above and dissipates. This dissipation of the flux rope during the eruption is similar to the findings reported by Liu et al. (2014b) and Chintzoglou et al. (2017). During the eruption, a cool erupting filament can be identified in emission proxy images, without any signature of flaring, as the eruption is short-lasting and weak.

The second eruption is more energetic than the first. At later stages of the simulation, more flux has emerged and more energy is available to be released come the second eruption. In addition, the continued expansion of the lobes reduces the field strength and tension of the strapping field, allowing the flux rope to build up and rise more. However, the most important element is that the low-lying field lines reconnecting to form the flux rope are more sheared, resulting in more effective reconnection. This results in a larger pre-eruptive flux rope. During the eruption, this results in a powerful and longer lasting outflow from the current sheet below the flux rope, which assists the upwards acceleration of the rope. At the same time, the reconnection at the current sheet below the flux rope produces lines that add significant flux into the erupting flux rope and increases its twist. This leads to the flux rope not being dissipated by the reconnection above it. Instead, the size of the flux rope is enhanced. Also, the strong and longer lasting downflows from the current sheet below the flux rope result in the flaring of the postreconnection arcade. The erupting flux rope can be identified in both hot and cooler temperatures as an flux rope in the corona in the shape of the number eight. The above-mentioned process is an important result of our numerical investigation, as many observations suggest that a flux rope can form or be enhanced during confined flares (e.g. Guo et al. 2012; Patsourakos et al. 2013; Tziotziou et al. 2013; Chintzoglou et al. 2015; James et al. 2017, 2018; Liu et al. 2018). Our numerical model is in very good agreement with these observations, providing important insight into the confined-flare-to-flux-rope scenario.

Flux emergence simulations have studied quadrupolar configurations by assuming the emergence of a single $\Omega$-loop weakly twisted or non-twisted flux tubes (Murray et al. 2006; Archontis et al. 2013; Syntelis et al. 2015), the emergence of two $\Omega$-loop segments of a highly twisted flux tube (Lee et al. 2015; Fang \& Fan 2015; Toriumi \& Takasao 2017), or the emergence of kink unstable flux tubes (e.g. Takasao et al. 2015; Toriumi \& Takasao 2017; Knizhnik et al. 2018). We briefly discuss some differences between these approaches. Takasao et al. (2015) noted that the kink unstable flux tubes and the two $\Omega$-loop segments lead to some significant differences (see Introduction). We now discuss the differences between the emergence of two $\Omega$-loop highly twisted flux tube segments against the emergence of a single $\Omega$-loop weakly twisted flux tube segment. Both cases can lead to the simultaneous emergence of two bipoles of similar fluxes at the photosphere, which are magnetically linked below the photosphere. Both cases eventually form a quadrupolar region with a strong PIL that is able to form a 
low-lying post-emergence flux rope (e.g. comparison between Archontis et al. 2013, and our simulation).

However, the two cases can potentially produce different dynamics. To examine these possibilities, we can make a comparison based on our knowledge of the emergence of a highly twisted single $\Omega$-loop. Such flux tubes can form a $\delta$-spot, flux ropes, and eruptions (e.g. Leake et al. 2014; Syntelis et al. 2017 , 2019a), and can induce stronger rotation at the photospheric polarities (Sturrock \& Hood 2016). Therefore, the emergence of two $\Omega$-loop segments of a highly twisted flux tube should induce more shear into the atmosphere by both the direct emergence of horizontal field and by the higher vorticity, in comparison to a single $\Omega$-loop weakly twisted flux tube of similar flux and radius. However, during the emergence of single $\Omega$-loop weakly twisted flux tube, the inner polarities spread more horizontally as they are less constrained by the azimuthal tension associated with the twist of the flux tube. Therefore, in this case, it is possible that the shear induced by horizontal shearing is more pronounced along a more elongated PIL. Another difference between the two models could arise at the self-PILs of the two bipoles. A single $\Omega$-loop highly twisted flux tube emerge to form a bipolar region with a strong (self) PIL that is capable of building up new flux ropes (e.g. Syntelis et al. 2017). Therefore, in principle, the emergence of two $\Omega$-loop segments of a highly twisted flux tube could form two filaments: one above each of the two self-PILs, similar to some observations (e.g. Chen et al. 2018). However, for our numerical set-up and during the run-time of the simulation, we did not find such a case, and thus, further numerical investigation is required. It is possible that the emergence of two more localised $\Omega$-loop segments (i.e. smaller $\lambda$ ) is needed to produce strong self-PILs capable of building up flux ropes above them. The self-PILs of the two bipoles in the cases of a single $\Omega$-loop weakly twisted flux tube are less strong and less shearing develops along them. Thus, flux ropes could be difficult, if not impossible, to form at these self-PILs, at least until the decay phase of the active region. In the future, we aim to compare quadrupolar regions of different subphotospheric origin to study in detail the differences in injection of free energy into the atmosphere, the location where it occurs, and any subsequent eruptivity.

Acknowledgements. This project has received funding from the Science and Technology Facilities Council (UK) through the consolidated grant ST/S000402/1. The authors acknowledge support by the Royal Society grant $\mathrm{RGF} / \mathrm{EA} / 180232$. This work was supported by computational time granted from the Greek Research and Technology Network (GRNET) in the National HPC facility - ARIS. The authors acknowledge support by the ERC synergy grant "The Whole Sun".

\section{References}

Acheson, D. J. 1979, Sol. Phys., 62, 23

An, J. M., \& Magara, T. 2013, ApJ, 773, 21

Antiochos, S. K., DeVore, C. R., \& Klimchuk, J. A. 1999, ApJ, 510, 485

Arber, T., Longbottom, A., Gerrard, C., \& Milne, A. 2001, J. Comput. Phys., 171,151

Archontis, V., \& Hood, A. W. 2012, A\&A, 537, A62

Archontis, V., \& Syntelis, P. 2019, Phil. Trans. R. Soc. A: Math. Phys. Eng. Sci., 377, 20180387

Archontis, V., \& Török, T. 2008, A\&A, 492, L35

Archontis, V., Moreno-Insertis, F., Galsgaard, K., Hood, A., \& O’Shea, E. 2004, A\&A, 426, 1047

Archontis, V., Hood, A. W., \& Tsinganos, K. 2013, ApJ, 778, 42

Baker, D., van Driel-Gesztelyi, L., Brooks, D. H., et al. 2019, ApJ, 875, 35

Chen, H., Duan, Y., Yang, J., Yang, B., \& Dai, J. 2018, ApJ, 869, 78

Chintzoglou, G., Patsourakos, S., \& Vourlidas, A. 2015, ApJ, 809, 34

Chintzoglou, G., Vourlidas, A., Savcheva, A., et al. 2017, ApJ, 843, 93
Chintzoglou, G., Zhang, J., Cheung, M. C. M., \& Kazachenko, M. 2019, ApJ, 871,67

Dalmasse, K., Pariat, E., Valori, G., Démoulin, P., \& Green, L. M. 2013, A\&A, 555, L6

Fan, Y. 2001, ApJ, 554, L111

Fan, Y., Zweibel, E. G., Linton, M. G., \& Fisher, G. H. 1999, ApJ, 521, 460

Fang, F., \& Fan, Y. 2015, ApJ, 806, 79

Georgoulis, M. K., Nindos, A., \& Zhang, H. 2019, Phil. Trans. R. Soc. A: Math. Phys. Eng. Sci., 377, 20180094

Guo, Y., Ding, M. D., Schmieder, B., Démoulin, P., \& Li, H. 2012, ApJ, 746, 17

Guo, J., Lin, J., \& Deng, Y. 2014, MNRAS, 441, 2208

Inoue, S., Shiota, D., Bamba, Y., \& Park, S.-H. 2018, ApJ, 867, 83

James, A. W., Green, L. M., Palmerio, E., et al. 2017, Sol. Phys., 292, 71

James, A. W., Valori, G., Green, L. M., et al. 2018, ApJ, 855, L16

Janvier, M., Aulanier, G., Bommier, V., et al. 2014, ApJ, 788, 60

Kazachenko, M. D., Fisher, G. H., Welsch, B. T., Liu, Y., \& Sun, X. 2015, ApJ, 811,16

Kliem, B., \& Török, T. 2006, Phys. Rev. Lett., 96, 255002

Knizhnik, K. J., Linton, M. G., \& DeVore, C. R. 2018, ApJ, 864, 89

Künzel, H. 1960, Astron. Nachr., 285, 271

Leake, J. E., Linton, M. G., \& Török, T. 2013, ApJ, 778, 99

Leake, J. E., Linton, M. G., \& Antiochos, S. K. 2014, ApJ, 787, 46

Lee, E. J., Archontis, V., \& Hood, A. W. 2015, ApJ, 798, L10

Linton, M. G., Fisher, G. H., Dahlburg, R. B., \& Fan, Y. 1999, ApJ, 522, 1190

Liu, C., Deng, N., Liu, R., et al. 2012, ApJ, 745, L4

Liu, Y. D., Richardson, J. D., Wang, C., \& Luhmann, J. G. 2014a, ApJ, 788, L28

Liu, R., Titov, V. S., Gou, T., et al. 2014b, ApJ, 790, 8

Liu, L., Cheng, X., Wang, Y., et al. 2018, ApJ, 867, L5

Lynch, B. J., Antiochos, S. K., DeVore, C. R., Luhmann, J. G., \& Zurbuchen, T. H. 2008, ApJ, 683, 1192

Manchester, IV, W., Gombosi, T., DeZeeuw, D., \& Fan, Y. 2004, ApJ, 610, 588

Mitra, P. K., Joshi, B., Prasad, A., Veronig, A. M., \& Bhattacharyya, R. 2018, ApJ, 869, 69

Moore, R. L., \& Roumeliotis, G. 1992, in IAU Colloq. 133: Eruptive Solar Flares, eds. Z. Svestka, B. V. Jackson, \& M. E. Machado (Berlin: Springer Verlag), Lect. Notes Phys., 399, 69

Moore, R. L., Sterling, A. C., Hudson, H. S., \& Lemen, J. R. 2001, ApJ, 552, 833

Murray, M. J., Hood, A. W., Moreno-Insertis, F., Galsgaard, K., \& Archontis, V. 2006, A\&A, 460, 909

Parker, E. N. 1955, ApJ, 121, 491

Patsourakos, S., Vourlidas, A., \& Stenborg, G. 2013, ApJ, 764, 125

Patsourakos, S., Georgoulis, M. K., Vourlidas, A., et al. 2016, ApJ, 817, 14

Priest, E. R., \& Forbes, T. G. 2002, A\&ARv, 10, 313

Qiu, J., \& Yurchyshyn, V. B. 2005, ApJ, 634, L121

Schrijver, C. J. 2007, ApJ, 655, L117

Schrijver, C. J. 2009, AdSpR, 43, 739

Schrijver, C. J., Aulanier, G., Title, A. M., Pariat, E., \& Delannée, C. 2011, ApJ, 738, 167

Sturrock, Z., \& Hood, A. W. 2016, A\&A, 593, A63

Sun, X., Hoeksema, J. T., Liu, Y., et al. 2012, ApJ, 748, 77

Syntelis, P., Archontis, V., Gontikakis, C., \& Tsinganos, K. 2015, A\&A, 584, A10

Syntelis, P., Gontikakis, C., Patsourakos, S., \& Tsinganos, K. 2016, A\&A, 588, A16

Syntelis, P., Archontis, V., \& Tsinganos, K. 2017, ApJ, 850, 95

Syntelis, P., Archontis, V., \& Tsinganos, K. 2019a, ApJ, 876, 61

Syntelis, P., Archontis, V., \& Hood, A. 2019b, ApJ, 874, 15

Takasao, S., Fan, Y., Cheung, M. C. M., \& Shibata, K. 2015, ApJ, 813, 112

Toriumi, S., \& Takasao, S. 2017, ApJ, 850, 39

Toriumi, S., Iida, Y., Kusano, K., Bamba, Y., \& Imada, S. 2014, Sol. Phys., 289, 3351

Toriumi, S., Schrijver, C. J., Harra, L. K., Hudson, H., \& Nagashima, K. 2017, ApJ, 834, 56

Török, T., \& Kliem, B. 2005, ApJ, 630, L97

Tziotziou, K., Georgoulis, M. K., \& Liu, Y. 2013, ApJ, 772, 115

van Ballegooijen, A. A., \& Martens, P. C. H. 1989, ApJ, 343, 971

Vemareddy, P., Ambastha, A., \& Maurya, R. A. 2012, ApJ, 761, 60

Vourlidas, A., Syntelis, P., \& Tsinganos, K. 2012, Sol. Phys., 280, 509

Wang, S., Liu, C., Liu, R., et al. 2012, ApJ, 745, L17

Wang, R., Liu, Y. D., Yang, Z., \& Hu, H. 2014, ApJ, 791, 84

Yang, S., Büchner, J., Skála, J., \& Zhang, H. 2018, A\&A, 613, A27

Zhang, H., Brandenburg, A., \& Sokoloff, D. D. 2016, ApJ, 819, 146

Zheng, J.-C., Yang, Z.-L., Guo, J.-P., et al. 2017, Res. Astron. Astrophys., 17, 081

Zhou, G. P., Tan, C. M., Su, Y. N., et al. 2019, ApJ, 873, 23

Zirin, H., \& Liggett, M. A. 1987, Sol. Phys., 113, 267 